\title{
Multi-component Wronskian solution to the
}

\section{Kadomtsev-Petviashvili equation}

\author{
TAO XU ${ }^{1}$, FU-Wei SuN ${ }^{2}$, Yi ZHANG ${ }^{1}$ AND JUAN Li ${ }^{3,4}$
}

1. College of Science, China University of Petroleum, Beijing 102249, China.

2. College of Science, North China University of Technology, Beijing 100041, China.

3. State Key Laboratory of Remote Sensing Science, Jointly Sponsored

by the Institute of Remote Sensing Applications of Chinese of Sciences

Academy and Beijing Normal University, Beijing 100101, China.

4. Demonstration Centre, Spaceborne Remote Sensing National Space

Administration, Beijing 100101, China.

E-mail address: xutodd@126.com (T. Xu)

\begin{abstract}
It is known that the Kadomtsev-Petviashvili (KP) equation can be decomposed into the first two members of the coupled Ablowitz-Kaup-Newell-Segur (AKNS) hierarchy by the binary nonlinearization of Lax pairs. In this paper, we construct the $N$-th iterated Darboux transformation (DT) for the second- and third-order $m$-coupled AKNS systems. By using together the $N$-th iterated DT and Cramer's rule, we find that the KPII equation has the unreduced multi-component Wronskian solution and the KPI equation admits a reduced multi-component Wronskian solution. In particular, based on the unreduced and reduced two-component Wronskians, we obtain two families of fully-resonant line-soliton solutions which contain arbitrary numbers of asymptotic solitons as $\boldsymbol{y} \rightarrow \mp \infty$ to the KPII equation, and the ordinary $\boldsymbol{N}$-soliton solution to the KPI equation. In addition, we find that the KPI line solitons propagating in parallel can exhibit the bound state at the moment of collision.
\end{abstract}




\section{Introduction}

In 1970, Kadomtsev and Petviashvili [1] derived the following (2+1)-dimensional nonlinear dispersive wave equation

$$
\left(-4 u_{t}+6 u u_{x}+u_{x x x}\right)_{x}+3 \sigma^{2} u_{y y}=0 \quad\left(\sigma^{2}= \pm 1\right),
$$

to study the stability of soliton solutions of the Korteweg-de Vries $(\mathrm{KdV})$ equation with respect to weak transverse perturbations. Eq. (1) is usually called the Kadomtsev-Petviashvili (KP) equation, where $\sigma^{2}=$ -1 and 1 represent the KPI and KPII equations, respectively. As a natural extension of the KdV equation in two dimensions, the KP equation (1) with both cases of $\sigma^{2}$ has arisen in various physical contexts, e.g., plasma physics [1], surface and internal water waves [2], etc. The KP equation (1) is a universal completely-integrable (2+1)-dimensional nonlinear evolution equation (NLEE) [3] and its many integrable properties have been revealed in the past forty years, including the Lax representation [4], lump solutions to the KPI equation [5], line-soliton solutions to the KPI and KPII equations [6], an infinite number of symmetries and conservation laws [7], Painlevé property [8], Bäcklund transformation [9], Darboux transformation (DT) [10], a recursion operator and bi-Hamiltonian structure [11, 12], and similarity reductions to the Painlevé-type ordinary differential equations [14] and integrable (1+1)-dimensional NLEEs [15].

In recent years, the KPII equation has attracted intensive attention [16-23] because a large variety of soliton solutions of this equation were overlook in the literature before 2003. It is known that the KPII equation admits the solution $u=2 \frac{\partial^{2}}{\partial x^{2}} \ln \tau(x, y, t)$ with the tau function $\tau(x, y, t)$ expressible in the Wronskian form [24]

$$
\tau(x, y, t)=\left|\begin{array}{cccc}
f_{1} & f_{2} & \cdots & f_{N} \\
\frac{\partial f_{1}}{\partial x} & \frac{\partial f_{2}}{\partial x} & \cdots & \frac{\partial f_{N}}{\partial x} \\
\vdots & \vdots & \ddots & \vdots \\
\frac{\partial^{N-1} f_{1}}{\partial x^{N-1}} & \frac{\partial^{N-1} f_{2}}{\partial x^{N-1}} & \cdots & \frac{\partial^{N-1} f_{N}}{\partial x^{N-1}}
\end{array}\right|
$$

where $\left\{f_{h}\right\}_{h=1}^{N}$ are a set of linearly-independent solutions of the linear system: $\sigma f_{y}=f_{x x}(\sigma= \pm 1)$, $f_{t}=f_{x x x}$. Ref. [17] has revealed a general family of multi-soliton solutions to the KPII equation by noting that the functions $\left\{f_{h}\right\}_{h=1}^{N}$ can be chosen as $f_{h}(x, y, t)=\sum_{l=1}^{M} a_{h l} \mathrm{e}^{\kappa_{l} x+\sigma \kappa_{l}^{2} y+\kappa_{l}^{3} t}(1 \leq h \leq N)$, where $M$ is an positive integer greater than $N$, the phase parameters $\left\{\kappa_{l}\right\}_{l=1}^{M}$ are distinct, the constant coefficients $a_{h l}$ $(1 \leq h \leq N ; 1 \leq l \leq M)$ define an $N \times M$ coefficient matrix $A:=\left(a_{h l}\right)$, which is required to be of full rank [i.e., $\operatorname{rank}(A)=N]$ and all of whose nonzero $N \times N$ minors must be sign definite.

If the matrix $A$ is in the reduced row-echelon form (RREF) and satisfies the irreducibility conditions that each column of $A$ contains at least one nonzero element and each row of $A$ contains at least one nonzero element in addition to the pivot (first nonzero) entry, the function $\tau$ in Eq. (2) can yield a multi-soliton configuration with $N_{+}=N$ asymptotic solitons as $y \rightarrow+\infty$ and $N_{-}=M-N$ asymptotic solitons as $y \rightarrow$ 
$-\infty$ [17]. Moreover, it has been indicated [18-20, 22, 25] that the classification problem of soliton solutions to the KPII equation can be solved from the viewpoint of algebraic geometry and combinatorics because each equivalence class of $(N, M-N)$-soliton solutions corresponds to a derangement (which is a permutation with no fixed point) on $\{1,2 \ldots, M\}$ with $N$ excedances $[19,20]$, and each RREF $N \times M$ matrix $A$ (all of whose maximal minors are non-negative) belongs to a unique totally non-negative (TNN) Grassmann cell which is a further decomposed cell of the Schubert cell of the TNN Grassmannian $\operatorname{Gr}_{N M}^{\mathrm{tnn}}[25,26]$. In addition, the initial value problem of the KPII equation with V-and X-shape initial waves consisting of two distinct line solitons has also been studied by the direct numeric simulation [20-23], which gives an explanation of the Mach reflection phenomena in shallow water [20, 22].

Unlike the work in Refs. [16-23], the present paper is going to construct the multi-component Wronskian solution to the KP equation (1) and explore the underlying soliton solutions. The motivation of this study comes from the observation in two aspects. First, the binary nonlinearization of two symmetric Lax pairs can decompose the KP equation (1) into the first two members of the coupled Ablowitz-Kaup-Newell-Segur (AKNS) hierarchy [27, 28]:

$$
\begin{aligned}
& p_{j, y}=-\sigma^{-1}\left(p_{j, x x}-2 \sum_{n=1}^{m} p_{n} q_{n} p_{j}\right) \quad(1 \leq j \leq m), \\
& q_{j, y}=\sigma^{-1}\left(q_{j, x x}-2 \sum_{n=1}^{m} q_{n} p_{n} q_{j}\right) \quad(1 \leq j \leq m),
\end{aligned}
$$

and

$$
\begin{aligned}
& p_{j, t}=p_{j, x x x}-3 \sum_{n=1}^{m} p_{n} q_{n} p_{j, x}-3 \sum_{n=1}^{m} p_{n, x} q_{n} p_{j} \quad(1 \leq j \leq m), \\
& q_{j, t}=q_{j, x x x}-3 \sum_{n=1}^{m} q_{n} p_{n} q_{j, x}-3 \sum_{n=1}^{m} q_{n, x} p_{n} q_{j}=0 \quad(1 \leq j \leq m),
\end{aligned}
$$

where $m$ is an arbitrary positive integer, Systems (3) and (4) are respectively referred to as the second- and third-order $m$-coupled AKNS systems, and

$$
u=-2 \sum_{j=1}^{m} p_{j} q_{j},
$$

exactly satisfies the KP equation (1). Second, the soliton solutions to both the vector nonlinear Schrödinger (NLS) equation and vector complex modified KdV equation, which are respectively two reductions of Systems (3) and (4), have been found to be expressible in terms of the multi-component Wronskian [29-31]. Therefore, it is reasonable to infer that the KP equation (1) also admits the multi-component Wronskian solution. The structure of this paper is organized as follows.

In Section 2, we follow the way in Ref. [32] to explicitly construct the $N$-th iterated DT for Systems (3) and (4), which provides a basis for us to further obtain the multi-component Wronskian solution to the KP 
equation (1). We note that Ref. [32] has presented a general scheme of constructing the $N$-th iterated DT for the AKNS hierarchy. However, there has been an absence of rigorous proof for such $N$-th iterated DT. In this section, we give a complete proof that the Lax pairs associated with Systems (3) and (4) keep the form-invariance under the $N$-th iterated DT.

In most of the previous literature (see, for example, Refs. [24, 33]), researchers usually obtain the Wronskian solutions by assuming that a given NLEE admits the Wronskian solution with certain condition, and then proving their conjecture by direct substitution the solution into the original equation or bilinear equation(s). In fact, one can also realize the Wronskian solutions to those Lax-integrable NLEEs by using together the $N$-th iterated DT algorithm and Cramer's rule rather than relying on the guesswork [31]. Moreover, if the Lax pair has been proved to be form-invariant under the DT, there is no need to verify the Wronskian solution again by means of the Plücker relation. In Section 3, we derive the multi-component Wronskian solution to the KP equation (1) by combining the $N$-th iterated DT and Cramer's rule.

In Section 4, we study the soliton solutions to the KP equation generated by the multi-component Wronskian. In Section 4.1, we from an unreduced two-component Wronskian derive two families of fullyresonant line-soliton solutions to the KPII equation, which are shown to be two special cases of the soliton solutions generated by the Wronskian (2). In Section 4.2, based on a reduced two-component Wronskian, we obtain the ordinary $N$-soliton solution to the KPI equation which, in general, describes the elastic collisions of line solitons. In particular, we find the bound state exhibited by the line solitons propagating in parallel at the moment of collision.

Finally, in Section 5, we address the conclusions of this paper.

\section{$2 \quad N$-th iterated Darboux transformation}

In the scheme of the $(m+1) \times(m+1)$-matrix AKNS scattering formulation [34], the Lax representation of Systems (3) and (4) can be written in the form

$$
\begin{aligned}
& \Phi_{x}=U(\lambda) \Phi=\left(\lambda U_{0}+U_{1}\right) \Phi, \\
& \Phi_{y}=V(\lambda) \Phi=\left(\lambda^{2} V_{0}+\lambda V_{1}+V_{2}\right) \Phi, \\
& \Phi_{t}=W(\lambda) \Phi=\left(\lambda^{3} W_{0}+\lambda^{2} W_{1}+\lambda W_{2}+W_{3}\right) \Phi,
\end{aligned}
$$

with

$$
\begin{aligned}
& U_{0}=\left(\begin{array}{cc}
1 & \mathbf{0} \\
\mathbf{0} & -E_{m}
\end{array}\right), \quad U_{1}=\left(\begin{array}{cc}
0 & \mathbf{p} \\
\mathbf{q}^{\top} & \mathbf{0}
\end{array}\right), \\
& V_{0}=-\frac{2}{\sigma} U_{0}, \quad V_{1}=-\frac{2}{\sigma} U_{1}, \quad V_{2}=\frac{1}{\sigma}\left(\begin{array}{cc}
\mathbf{p} \mathbf{q}^{\top} & -\mathbf{p}_{x} \\
\mathbf{q}_{x}^{\top} & -\mathbf{q}^{\top} \mathbf{p}
\end{array}\right),
\end{aligned}
$$




$$
\begin{aligned}
& W_{0}=4 U_{0}, \quad W_{1}=4 U_{1}, \quad W_{2}=-2 \sigma V_{2}, \\
& W_{3}=\left(\begin{array}{cc}
\mathbf{p q}_{x}^{\top}-\mathbf{p}_{x} \mathbf{q}^{\top} & \mathbf{p}_{x x}-2 \mathbf{p} \mathbf{q}^{\top} \mathbf{p} \\
\mathbf{q}_{x x}^{\top}-2 \mathbf{q}^{\top} \mathbf{p} \mathbf{q}^{\top} & \mathbf{q}^{\top} \mathbf{p}_{x}-\mathbf{q}_{x}^{\top} \mathbf{p}
\end{array}\right),
\end{aligned}
$$

where $\lambda$ is the spectral parameter, $\Phi=\left(\phi_{1}, \phi_{2}, \ldots, \phi_{m+1}\right)^{\top}$ ( $\top$ denotes the transpose of vector) is the vector eigenfunction, $\mathbf{p}=\left(p_{1}, p_{2}, \ldots, p_{m}\right)$ and $\mathbf{q}=\left(q_{1}, q_{2}, \ldots, q_{m}\right)$ are the vector potentials, $E_{m}$ is the $m \times m$ identity matrix, and the compatibility conditions $\Phi_{x y}=\Phi_{y x}$ and $\Phi_{x t}=\Phi_{t x}$ yield Systems (3) and (4), respectively.

According to the idea of the DT [32], it requires that under the $N$-th iterated eigenfunction transformation

$$
\Phi^{\prime}=T(\lambda) \Phi
$$

the new eigenfunction $\Phi^{\prime}=\left(\phi_{1}^{\prime}, \phi_{2}^{\prime}, \ldots, \phi_{m+1}^{\prime}\right)^{\top}$ also satisfies System (6) with the matrices $U(\lambda), V(\lambda)$ and $W(\lambda)$ replaced respectively by $U^{\prime}(\lambda), V^{\prime}(\lambda)$ and $W^{\prime}(\lambda)$ in which the new potentials $\mathbf{p}^{\prime}=\left(p_{1}^{\prime}, p_{2}^{\prime}, \ldots, p_{m}^{\prime}\right)$ and $\mathbf{q}^{\prime}=\left(q_{1}^{\prime}, q_{2}^{\prime}, \ldots, q_{m}^{\prime}\right)$ are respectively instead of the old potentials $\mathbf{p}$ and $\mathbf{q}$, that is,

$$
\Phi_{x}^{\prime}=U^{\prime}(\lambda) \Phi^{\prime}, \quad \Phi_{y}^{\prime}=V^{\prime}(\lambda) \Phi^{\prime}, \quad \Phi_{t}^{\prime}=W^{\prime}(\lambda) \Phi^{\prime} .
$$

Thus, the Darboux matrix $T(\lambda)$ has to obey the following three conditions:

$$
\begin{aligned}
& T_{x}(\lambda)+T(\lambda) U(\lambda)=U^{\prime}(\lambda) T(\lambda), \\
& T_{y}(\lambda)+T(\lambda) V(\lambda)=V^{\prime}(\lambda) T(\lambda), \\
& T_{t}(\lambda)+T(\lambda) W(\lambda)=W^{\prime}(\lambda) T(\lambda),
\end{aligned}
$$

where Conditions (9a)-(9c) respectively correspond to the form-invariance of Eqs. (6a)-(6c).

For the satisfaction of Conditions (9a)-(9c), we assume that the matrix $T(\lambda)$ is of the form [32]

$$
T(\lambda)=\left(\begin{array}{cccc}
\alpha(\lambda) & \beta_{1}(\lambda) & \cdots & \beta_{m}(\lambda) \\
\gamma_{1}(\lambda) & \delta_{11}(\lambda) & \cdots & \delta_{1 m}(\lambda) \\
\vdots & \vdots & \ddots & \vdots \\
\gamma_{m}(\lambda) & \delta_{m 1}(\lambda) & \cdots & \delta_{m m}(\lambda)
\end{array}\right)
$$

with

$$
\begin{aligned}
& \alpha(\lambda)=\lambda^{N}-\sum_{n=0}^{N-1} \alpha^{(n)} \lambda^{n}, \quad \beta_{j}(\lambda)=\sum_{n=0}^{N-1} \beta_{j}^{(n)}(-\lambda)^{n} \quad(1 \leq j \leq m), \\
& \gamma_{i}(\lambda)=-\sum_{n=0}^{N-1} \gamma_{i}^{(n)} \lambda^{n} \quad(1 \leq i \leq m), \quad \delta_{i i}(\lambda)=\lambda^{N}+\sum_{n=0}^{N-1} \delta_{i i}^{(n)}(-\lambda)^{n} \quad(1 \leq i \leq m), \\
& \delta_{i j}(\lambda)=\sum_{n=0}^{N-1} \delta_{i j}^{(n)}(-\lambda)^{n} \quad(1 \leq i, j \leq m ; i \neq j),
\end{aligned}
$$


where $\beta_{j}^{(n)}, \gamma_{i}^{(n)}$ and $\delta_{i j}^{(n)}(1 \leq i, j \leq m ; 0 \leq n \leq N-1)$ are the functions of $x, y$ and $t$ which are determined by

$$
T\left(\lambda_{k}\right) \Phi_{k}=\mathbf{0} \quad(1 \leq k \leq K=m N+N),
$$

with $\Phi_{k}=\left(f_{k}, g_{k}^{(1)}, \ldots, g_{k}^{(m)}\right)^{\top}$ as the solution of System (6) corresponding to $\lambda=\lambda_{k}\left(\lambda_{k} \neq \lambda_{l} ; 1 \leq\right.$ $k, l \leq K)$. It should be noted that $\left\{\Phi_{k}\right\}_{k=1}^{K}$ are a set of linearly-independent solutions of System (6) because $\lambda_{k} \neq \lambda_{l}$. Accordingly, one can uniquely determine $\beta_{j}^{(n)}, \gamma_{i}^{(n)}$ and $\delta_{i j}^{(n)}(1 \leq i, j \leq m ; 0 \leq n \leq N-1)$ from (14) which can be expanded as

$$
\alpha\left(\lambda_{k}\right)+\sum_{j=1}^{m} \varpi_{k}^{(j)} \beta_{j}\left(\lambda_{k}\right)=0, \quad \gamma_{i}\left(\lambda_{k}\right)+\sum_{j=1}^{m} \varpi_{k}^{(j)} \delta_{i j}\left(\lambda_{k}\right)=0 \quad(1 \leq i \leq m ; 1 \leq k \leq K),
$$

where $\varpi_{k}^{(j)}=g_{k}^{(j)} / f_{k}(1 \leq j \leq m ; 1 \leq k \leq K)$.

Before proving that Conditions (9a)-(9c) are satisfied with the Darboux matrix $T(\lambda)$ given by Eq. (10), in which $\beta_{j}^{(n)}, \gamma_{i}^{(n)}$ and $\delta_{i j}^{(n)}(1 \leq i, j \leq m ; 0 \leq n \leq N-1)$ are determined by Eqs. (15), we present the following two important lemmas.

Lemma 2.1. Let the Darboux matrix $T(\lambda)$ be in the form of $(10)$ with $\beta_{j}^{(n)}, \gamma_{i}^{(n)}$ and $\delta_{i j}^{(n)}(1 \leq i, j \leq m$; $0 \leq n \leq N-1$ ) determined by Eqs. (15). Then, the determinant of $T(\lambda)$ can be expanded as

$$
\operatorname{det} T(\lambda)=\prod_{k=1}^{K}\left(\lambda-\lambda_{k}\right)
$$

where $\lambda_{k}(1 \leq k \leq K)$ are the roots of $\operatorname{det} T(\lambda)$.

Proof. It is easy to know that $\operatorname{det} T(\lambda)$ is a monic polynomial of degree $K$. On the other hand, one can employ Eqs. (15) to express the first column of $T\left(\lambda_{k}\right)$ as the linear combination of other columns:

$$
\left[\alpha\left(\lambda_{k}\right), \gamma_{1}\left(\lambda_{k}\right), \ldots, \gamma_{m}\left(\lambda_{k}\right)\right]^{\top}=-\sum_{j=1}^{m} \varpi_{k}^{(j)}\left[\beta_{j}\left(\lambda_{k}\right), \delta_{1 j}\left(\lambda_{k}\right), \ldots, \delta_{m j}\left(\lambda_{k}\right)\right]^{\top} \quad(1 \leq k \leq K),
$$

which suggests that $\lambda_{k}(1 \leq k \leq K)$ are the roots of $\operatorname{det} T(\lambda)$. Therefore, the determinant of $T(\lambda)$ can be expressed in the form of (16).

\section{Lemma 2.2. Let us define that}

$$
\begin{aligned}
& {\left[u_{h l}(\lambda)\right]_{(m+1) \times(m+1)}=\left[T_{x}(\lambda)+T(\lambda) U(\lambda)\right] T^{*}(\lambda),} \\
& {\left[v_{h l}(\lambda)\right]_{(m+1) \times(m+1)}=\left[T_{y}(\lambda)+T(\lambda) V(\lambda)\right] T^{*}(\lambda),} \\
& {\left[w_{h l}(\lambda)\right]_{(m+1) \times(m+1)}=\left[T_{t}(\lambda)+T(\lambda) W(\lambda)\right] T^{*}(\lambda),}
\end{aligned}
$$


where $T^{*}(\lambda)$ is the adjoint matrix of $T(\lambda)$. Then, $\lambda_{k}(1 \leq k \leq K)$ are the roots of $u_{h l}(\lambda)$, $v_{h l}(\lambda)$ and $w_{h l}(\lambda)(1 \leq h, l \leq m+1)$, that is,

$$
u_{h l}\left(\lambda_{k}\right)=0, \quad v_{h l}\left(\lambda_{k}\right)=0, \quad w_{h l}\left(\lambda_{k}\right)=0 \quad(1 \leq h, l \leq m+1 ; 1 \leq k \leq K) .
$$

The proof of Lemma 2.2 is given in Appendix A. In the following, we use $\operatorname{deg}[f(\lambda)]$ to represent the degree of the polynomial $f(\lambda)$, and $\alpha^{*}(\lambda), \beta_{j}^{*}(\lambda), \gamma_{i}^{*}(\lambda)$ and $\delta_{i j}^{*}(\lambda)$ to denote the algebraic cofactors of $\alpha(\lambda), \beta_{j}(\lambda), \gamma_{i}(\lambda)$ and $\delta_{i j}(\lambda)(1 \leq i, j \leq m)$, respectively.

Proposition 2.3. Assume that $\Phi_{k}=\left(f_{k}, g_{k}^{(1)}, \ldots, g_{k}^{(m)}\right)^{\top}$ satisfies Eq. (6a) with $\lambda=\lambda_{k}$, where $1 \leq k \leq K$. Then, the Darboux matrix $T(\lambda)$ given by (10) obeys Condition $(9 a)$, provided that $\beta_{j}^{(n)}, \gamma_{i}^{(n)}$ and $\delta_{i j}^{(n)}$ $(1 \leq i, j \leq m ; 0 \leq n \leq N-1)$ are determined by Eqs. (15), and the $N$-th iterated potential transformations are given by

$$
\mathbf{p}^{\prime}=\mathbf{p}-2(-1)^{N-1} \mathbf{b}^{(N-1)}, \quad \mathbf{q}^{\prime}=\mathbf{q}-2 \mathbf{c}^{(N-1)},
$$

where $\mathbf{b}^{(N-1)}=\left(\beta_{1}^{(N-1)}, \ldots, \beta_{m}^{(N-1)}\right)$ and $\mathbf{c}^{(N-1)}=\left(\gamma_{1}^{(N-1)}, \ldots, \gamma_{m}^{(N-1)}\right)$.

Proof. Let $\Pi(\lambda)=\left[T_{x}(\lambda)+T(\lambda) U(\lambda)\right] T^{*}(\lambda) / \operatorname{det} T(\lambda)$. The calculation of algebraic cofactors $\alpha^{*}(\lambda)$, $\beta_{j}^{*}(\lambda), \gamma_{i}^{*}(\lambda)$ and $\delta_{i j}^{*}(\lambda)$ yields that $\operatorname{deg}\left[\alpha^{*}(\lambda)\right]=\operatorname{deg}\left[\delta_{i i}^{*}(\lambda)\right]=m N$ and $\operatorname{deg}\left[\beta_{j}^{*}(\lambda)\right]=\operatorname{deg}\left[\gamma_{i}^{*}(\lambda)\right]=$ $\operatorname{deg}\left[\delta_{i j}^{*}(\lambda)\right]=m N-1(1 \leq i, j \leq m ; i \neq j)$, which tells us that $\operatorname{deg}\left[u_{h h}(\lambda)\right]=K+1$ and $\operatorname{deg}\left[u_{h l}(\lambda)\right]=K$ $(1 \leq h, l \leq m+1 ; h \neq l)$. On the other hand, Lemmas 2.1 and 2.2 imply that $u_{h l}(\lambda)(1 \leq h, l \leq m+1)$ can be exactly divided by $\operatorname{det} T(\lambda)$. Therefore, the matrix $\Pi(\lambda)$ can be written in the form

$$
\Pi(\lambda)=\left(\begin{array}{ccccc}
\lambda \pi_{11}^{(1)}+\pi_{11}^{(0)} & \pi_{12}^{(0)} & \ldots & \pi_{1, m+1}^{(0)} \\
\pi_{21}^{(0)} & \lambda \pi_{22}^{(1)}+\pi_{22}^{(0)} & \ldots & \pi_{2, m+1}^{(0)} \\
\vdots & \vdots & \ddots & \vdots \\
\pi_{m+1,1}^{(0)} & \pi_{m+1,2}^{(0)} & \ldots & \lambda \pi_{m+1, m+1}^{(1)}+\pi_{m+1, m+1}^{(0)}
\end{array}\right)
$$

where $\pi_{h h}^{(1)}$ and $\pi_{h l}^{(0)}(1 \leq h, l \leq m+1)$ are all the functions dependent on $x, y$ and $t$. By expanding $T_{x}(\lambda)+T(\lambda) U(\lambda)=\Pi(\lambda) T(\lambda)$ and comparing the coefficients of $\lambda^{N+1}$ and $\lambda^{N}$, we can find

$$
\begin{aligned}
& \pi_{11}^{(1)}=1, \quad \pi_{11}^{(0)}=0, \quad \pi_{i+1, i+1}^{(1)}=-1, \quad \pi_{i+1, j+1}^{(0)}=0 \quad(1 \leq i, j \leq m), \\
& \pi_{1, j+1}^{(0)}=p_{j}-2(-1)^{N-1} \beta_{j}^{(N-1)}, \quad \pi_{i+1,1}^{(0)}=q_{i}-2 \gamma_{i}^{(N-1)} \quad(1 \leq i, j \leq m) .
\end{aligned}
$$

It is obvious that the matrix $\Pi(\lambda)$ is exactly equal to $U^{\prime}(\lambda)$ with the new potentials $\mathbf{p}^{\prime}$ and $\mathbf{q}^{\prime}$ given by (20), which means that $T_{x}(\lambda)+T(\lambda) U(\lambda)=U^{\prime}(\lambda) T(\lambda)$.

Similarly, we present other two propositions, as stated in the following: 
Proposition 2.4. Assume that $\Phi_{k}=\left(f_{k}, g_{k}^{(1)}, \ldots, g_{k}^{(m)}\right)^{\top}$ satisfies Eq. (6b) with $\lambda=\lambda_{k}$, where $1 \leq k \leq K$. Then, the Darboux matrix $T(\lambda)$ in (10) obeys Condition (9b), provided that $\beta_{j}^{(n)}, \gamma_{i}^{(n)}$ and $\delta_{i j}^{(n)}(1 \leq i, j \leq$ $m ; 0 \leq n \leq N-1$ ) are determined by Eqs. (15), and the new potentials $\mathbf{p}^{\prime}$ and $\mathbf{q}^{\prime}$ are given by (20).

Proposition 2.5. Assume that $\Phi_{k}=\left(f_{k}, g_{k}^{(1)}, \ldots, g_{k}^{(m)}\right)^{\top}$ satisfies Eq. (6c) with $\lambda=\lambda_{k}$, where $1 \leq k \leq K$. Then, the Darboux matrix $T(\lambda)$ in (10) obeys Condition (9c), provided that $\beta_{j}^{(n)}, \gamma_{i}^{(n)}$ and $\delta_{i j}^{(n)}(1 \leq i, j \leq$ $m ; 0 \leq n \leq N-1$ ) are determined by Eqs. (15), and the new potentials $\mathbf{p}^{\prime}$ and $\mathbf{q}^{\prime}$ are given by (20).

Seen from Propositions 2.3-2.5, the Darboux matrix $T(\lambda)$ makes sure that the new eigenfunction $\Phi^{\prime}=$ $T(\lambda) \Phi$ also satisfies Lax representation (6) for the new potentials $\mathbf{p}^{\prime}$ and $\mathbf{q}^{\prime}$ given by (20). That is to say, the compatibility conditions $\Phi_{x y}^{\prime}=\Phi_{y x}^{\prime}$ and $\Phi_{x t}^{\prime}=\Phi_{t x}^{\prime}$ give the same systems (3) and (4) except for $\mathbf{p}^{\prime}$ and $\mathbf{q}^{\prime}$ instead of $\mathbf{p}$ and $\mathbf{q}$, respectively. Therefore, we come to the following theorem:

Theorem 2.6. Suppose that $\left\{\Phi_{k}\right\}_{k=1}^{K}$ are a set of linearly-independent solutions of System (6) which correspond to a set of different spectral parameters $\left\{\lambda_{k}\right\}_{k=1}^{K}$. The eigenfunction transformation (7) and the potential transformations (20) constitute the $N$-th iterated $D T(\Phi, \mathbf{q}, \mathbf{p}) \rightarrow\left(\Phi^{\prime}, \mathbf{q}^{\prime}, \mathbf{p}^{\prime}\right)$ of Systems (3) and (4), where the Darboux matrix $T(\lambda)$ is determined by Eqs. (15).

Remark 2.7. With some constraints between the potentials $\mathbf{p}$ and $\mathbf{q}$, Systems (3) and (4) can be reduced to the NLEEs belonging to some known integrable hierarchies such as the coupled NLS hierarchy [35] $(\mathbf{p}= \pm \overline{\mathbf{q}}$, where the bar represents complex conjugate) and coupled modified KdV hierarchy [36] $(\mathbf{p}= \pm \mathbf{q})$. In the above way, one can also use Transformations (7) and (20) to constitute the $N$-th iterated DT for those reduced cases, but $\left\{\Phi_{k}\right\}_{k=1}^{K}$ are required to be particularly selected. For example, if $\mathbf{p}= \pm \overline{\mathbf{q}}$, the functions $\left\{\Phi_{k}\right\}_{k=1}^{K}$ are chosen as [31]

$$
\begin{aligned}
& \Phi_{(k-1)(m+1)+1}=\left(f_{k}, g_{k}^{(1)}, \ldots, g_{k}^{(m)}\right)^{\top} \quad(1 \leq k \leq N), \\
& \Phi_{(k-1)(m+1)+j+1}=( \pm \bar{g}_{k}^{(j)}, \overbrace{0, \ldots, 0}^{j-1}, \bar{f}_{k}, \overbrace{0, \ldots, 0}^{m-j})^{\top} \quad(1 \leq k \leq N ; 1 \leq j \leq m),
\end{aligned}
$$

where $\Phi_{(k-1)(m+1)+1}$ satisfies System (6) with $\mathbf{p}= \pm \overline{\mathbf{q}}$ and $\lambda=\lambda_{k}(1 \leq k \leq N)$, and $\Phi_{(k-1)(m+1)+j+1}$ $(1 \leq j \leq m)$ are orthogonal to $\Phi_{(k-1)(m+1)+1}$.

\section{Multi-component Wronskian solution}

In this section, by combining the $N$-th iterated DT of Systems (3) and (4) and integrable decomposition from the KP equation (1) to Systems (3) and (4), we try to construct the multi-component Wronskian solution to the KP equation (1). 
In order to solve the functions $\beta_{j}^{(N-1)}$ and $\gamma_{i}^{(N-1)}(1 \leq i, j \leq m)$, we rewrite Eqs. (15) in the following form

$$
\begin{aligned}
& \left(F_{K \times N},-G_{K \times N}^{(1)}, \cdots,-G_{K \times N}^{(m)}\right) \mathbf{X}=\left(\lambda_{1}^{N} f_{1}, \cdots, \lambda_{K}^{N} f_{K}\right)^{\top} \\
& \left(F_{K \times N},-G_{K \times N}^{(1)}, \cdots,-G_{K \times N}^{(m)}\right) \mathbf{Y}_{i}=\left(\lambda_{1}^{N} g_{1}^{(i)}, \cdots, \lambda_{K}^{N} g_{K}^{(i)}\right)^{\top} \quad(1 \leq i \leq m),
\end{aligned}
$$

where

$$
\begin{aligned}
& F_{K \times N}=\left(\begin{array}{cccc}
f_{1} & \lambda_{1} f_{1} & \cdots & \lambda_{1}^{N-1} f_{1} \\
f_{2} & \lambda_{2} f_{2} & \cdots & \lambda_{2}^{N-1} f_{2} \\
\vdots & \vdots & \ddots & \vdots \\
f_{K} & \lambda_{K} f_{K} & \cdots & \lambda_{K}^{N-1} f_{K}
\end{array}\right), \\
& G_{K \times N}^{(j)}=\left(\begin{array}{cccc}
g_{1}^{(j)} & -\lambda_{1} g_{1}^{(j)} & \cdots & \left(-\lambda_{1}\right)^{N-1} g_{1}^{(j)} \\
g_{2}^{(j)} & -\lambda_{2} g_{2}^{(j)} & \cdots & \left(-\lambda_{2}\right)^{N-1} g_{2}^{(j)} \\
\vdots & \vdots & \ddots & \vdots \\
g_{K}^{(j)} & -\lambda_{K} g_{K}^{(j)} & \cdots & \left(-\lambda_{K}\right)^{N-1} g_{K}^{(j)}
\end{array}\right) \quad(1 \leq j \leq m), \\
& \mathbf{X}=\left(\alpha^{(0)}, \alpha^{(1)}, \ldots, \alpha^{(N-1)} ; \beta_{1}^{(0)}, \beta_{1}^{(1)}, \ldots, \beta_{1}^{(N-1)} ; \ldots ; \beta_{m}^{(0)}, \beta_{m}^{(1)}, \ldots, \beta_{m}^{(N-1)}\right)^{\top}, \\
& \mathbf{Y}_{i}=\left(\gamma_{i}^{(0)}, \gamma_{i}^{(1)}, \ldots, \gamma_{i}^{(N-1)} ; \delta_{i 1}^{(0)}, \delta_{i 1}^{(1)}, \ldots, \delta_{i 1}^{(N-1)} ; \ldots ; \delta_{i m}^{(0)}, \delta_{i m}^{(1)}, \ldots, \delta_{i m}^{(N-1)}\right)^{\top} \quad(1 \leq i \leq m) .
\end{aligned}
$$

By employing Cramer's rule, we can obtain the functions $\beta_{j}^{(N-1)}$ and $\gamma_{i}^{(N-1)}$ as

$$
\beta_{j}^{(N-1)}=(-1)^{j N-1} \frac{\chi_{j}^{(1)}}{\tau}, \quad \gamma_{i}^{(N-1)}=(-1)^{(i-1) N-1} \frac{\chi_{i}^{(2)}}{\tau} \quad(1 \leq i, j \leq m),
$$

with

$$
\begin{aligned}
& \tau=\operatorname{det}\left(F_{K \times N},-G_{K \times N}^{(1)}, \cdots,-G_{K \times N}^{(m)}\right), \\
& \chi_{j}^{(1)}=\operatorname{det}\left(F_{K \times(N+1)},-G_{K \times N}^{(1)}, \cdots,-G_{K \times(N-1)}^{(j)}, \cdots,-G_{K \times N}^{(m)}\right) \quad(1 \leq j \leq m), \\
& \chi_{i}^{(2)}=\operatorname{det}\left(F_{K \times(N-1)},-G_{K \times N}^{(1)}, \cdots,-G_{K \times(N+1)}^{(i)}, \cdots,-G_{K \times N}^{(m)}\right) \quad(1 \leq i \leq m) .
\end{aligned}
$$

where $\tau, \chi_{j}^{(1)}$ and $\chi_{i}^{(2)}(1 \leq i, j \leq m)$ are the multi-component Wronskians which have been proposed in Ref. [29]. Thus, the $N$-th iterated potential transformations (20) can be written as

$$
p_{j}^{\prime}=p_{j}+2(-1)^{(j+1) N+1} \frac{\chi_{j}^{(1)}}{\tau}, \quad q_{i}^{\prime}=q_{i}-2(-1)^{(i-1) N-1} \frac{\chi_{i}^{(2)}}{\tau} \quad(1 \leq i, j \leq m) .
$$

With $\mathbf{p}=\mathbf{q}=0$, the general solution of System (6) with $\lambda=\lambda_{k}\left(\lambda_{k} \neq \lambda_{l} ; 1 \leq k, l \leq K\right)$ is given as follows:

$$
\Phi_{k}=\left(f_{k}, g_{k}^{(1)}, \ldots, g_{k}^{(m)}\right)^{\top}=\left(a_{k} \mathrm{e}^{\frac{1}{2} \theta_{k}}, b_{k}^{(1)} \mathrm{e}^{-\frac{1}{2} \theta_{k}}, \ldots, b_{k}^{(m)} \mathrm{e}^{-\frac{1}{2} \theta_{k}}\right)^{\top} \quad(1 \leq k \leq K),
$$


where

$$
\theta_{k}=2\left(\lambda_{k} x-2 \sigma \lambda_{k}^{2} y+4 \lambda_{k}^{3} t\right) .
$$

It is noted that the field $u$ of Eq. (1) is usually assumed to be a real one. For the case $\sigma^{2}=1$ which corresponds to the KPII equation, we require the parameters $\lambda_{k}, a_{k}$ and $b_{k}^{(j)}(1 \leq k \leq K ; 1 \leq j \leq m)$ be all real constants.

Proposition 3.1. With $\Phi_{k}=\left(f_{k}, g_{k}^{(1)}, \ldots, g_{k}^{(m)}\right)^{\top}$ given by (28) for $1 \leq k \leq K$, the KPII equation admits the solution $u=2(\ln \tau)_{x x}$, where the tau function $\tau$ is expressed as the following unreduced multicomponent Wronskian:

$$
\tau=\left|A_{K \times K} \Theta_{K \times K}^{+} \Lambda_{K \times N}^{+}, B_{K \times K}^{(1)} \Theta_{K \times K}^{-} \Lambda_{K \times N}^{-}, \ldots, B_{K \times K}^{(m)} \Theta_{K \times K}^{-} \Lambda_{K \times N}^{-}\right|,
$$

with

$$
\begin{aligned}
& A_{K \times K}=\operatorname{diag}\left(a_{1}, \ldots, a_{K}\right), \quad B_{K \times K}^{(j)}=\operatorname{diag}\left(-b_{1}^{(j)}, \ldots,-b_{K}^{(j)}\right) \quad(1 \leq j \leq m), \\
& \Theta_{K \times K}^{+}=\operatorname{diag}\left(\mathrm{e}^{\frac{1}{2} \theta_{1}}, \ldots, \mathrm{e}^{\frac{1}{2} \theta_{K}}\right), \quad \Theta_{K \times K}^{-}=\operatorname{diag}\left(\mathrm{e}^{-\frac{1}{2} \theta_{1}}, \ldots, \mathrm{e}^{-\frac{1}{2} \theta_{K}}\right), \\
& \Lambda_{K \times N}^{+}=\left(\begin{array}{cccc}
1 & \lambda_{1} & \cdots & \lambda_{1}^{N-1} \\
1 & \lambda_{2} & \cdots & \lambda_{2}^{N-1} \\
\vdots & \vdots & \ddots & \vdots \\
1 & \lambda_{K} & \cdots & \lambda_{K}^{N-1}
\end{array}\right), \quad \Lambda_{K \times N}^{-}=\left(\begin{array}{cccc}
1 & -\lambda_{1} & \cdots & \left(-\lambda_{1}\right)^{N-1} \\
1 & -\lambda_{2} & \cdots & \left(-\lambda_{2}\right)^{N-1} \\
\vdots & \vdots & \ddots & \vdots \\
1 & -\lambda_{K} & \cdots & \left(-\lambda_{K}\right)^{N-1}
\end{array}\right) .
\end{aligned}
$$

Proof. With $\mathbf{p}=\mathbf{q}=0$, substituting Eqs. (27) into Eq. (5) yields

$$
u=-2 \sum_{j=1}^{m} p_{j}^{\prime} q_{j}^{\prime}=8 \sum_{j=1}^{m} \frac{\chi_{j}^{(1)} \chi_{j}^{(2)}}{\tau^{2}} .
$$

On the other hand, one can follow part (ii) of Theorem 3.3 in Ref. [29] to obtain the following multicomponent Wronskian identity:

$$
\sum_{j=1}^{m} \chi_{j}^{(1)} \chi_{j}^{(2)}=\frac{1}{4}\left(\tau \tau_{x x}-\tau_{x}^{2}\right),
$$

which is substituted into the right-hand side of Eq. (31), giving that $u=2(\ln \tau)_{x x}$.

In the case $\sigma^{2}=-1$, the solution $u=2(\ln \tau)_{x x}$ with $\tau$ given by (30) also satisfies the KPI equation, but it does not meet the requirement that $u$ must be a real field. According to Remark 2.7, if imposing the constraint $\mathbf{p}=\varepsilon \overline{\mathbf{q}}(\varepsilon= \pm 1)$ on Systems (3) and (4), one can choose $\left\{\Phi_{k}\right\}_{k=1}^{K}$ as

$$
\begin{aligned}
& \Phi_{(k-1)(m+1)+1}=\left(a_{k} \mathrm{e}^{\frac{1}{2} \theta_{k}}, b_{k}^{(1)} \mathrm{e}^{-\frac{1}{2} \theta_{k}}, \ldots, b_{k}^{(m)} \mathrm{e}^{-\frac{1}{2} \theta_{k}}\right)^{\top} \quad(1 \leq k \leq N), \\
& \Phi_{(k-1)(m+1)+j+1}=(\varepsilon \bar{b}_{k}^{(j)} \mathrm{e}^{-\frac{1}{2} \bar{\theta}_{k}}, \overbrace{0, \ldots, 0}^{j-1}, \bar{a}_{k} \mathrm{e}^{\frac{1}{2} \bar{\theta}_{k}}, \overbrace{0, \ldots, 0}^{m-j})^{\top} \quad(1 \leq k \leq N ; 1 \leq j \leq m),
\end{aligned}
$$


where the phase $\theta_{k}$ is the same as given in Eq. (29), but the parameters $\lambda_{k}, a_{k}$ and $b_{k}^{(j)}(1 \leq k \leq N$; $1 \leq j \leq m)$ are all complex constants. Then, $p_{j}^{\prime}$ and its complex conjugate $\bar{p}_{j}^{\prime}$ are obtained as follows:

$$
p_{j}^{\prime}=2(-1)^{(j+1) N+1} \frac{\chi_{j}^{(1)}}{\tau}, \quad \bar{p}_{j}^{\prime}=2 \varepsilon(-1)^{(j-1) N} \frac{\chi_{j}^{(2)}}{\tau} \quad(1 \leq j \leq m) .
$$

where the functions $\tau, \chi_{j}^{(1)}$ and $\chi_{j}^{(2)}$ are given by

$$
\tau=\left|\begin{array}{cccc}
F_{N \times N} & \varepsilon G_{N \times N}^{(1)} & \cdots & \varepsilon G_{N \times N}^{(m)} \\
\bar{G}_{N \times N}^{(1)} & \bar{F}_{N \times N} & \cdots & \mathbf{0} \\
\vdots & \vdots & \ddots & \vdots \\
\bar{G}_{N \times N}^{(m)} & \mathbf{0} & \cdots & \bar{F}_{N \times N}
\end{array}\right|
$$

$$
\chi_{j}^{(1)}=\left|\begin{array}{cccccc}
F_{N \times(N+1)} & \varepsilon G_{N \times N}^{(1)} & \cdots & \varepsilon G_{N \times(N-1)}^{(j)} & \cdots & \varepsilon G_{N \times N}^{(m)} \\
\bar{G}_{N \times(N+1)}^{(1)} & \bar{F}_{N \times N} & \cdots & \mathbf{0} & \cdots & \mathbf{0} \\
\vdots & \vdots & \ddots & \vdots & \ddots & \vdots \\
\bar{G}_{N \times(N+1)}^{(j)} & \mathbf{0} & \cdots & \bar{F}_{N \times(N-1)} & \cdots & \mathbf{0} \\
\vdots & \vdots & \ddots & \vdots & \ddots & \vdots \\
\bar{G}_{N \times(N+1)}^{(m)} & \mathbf{0} & \cdots & \mathbf{0} & \cdots & \bar{F}_{N \times N}
\end{array}\right| \quad(1 \leq j \leq m),
$$

$$
\chi_{j}^{(2)}=\left|\begin{array}{cccccc}
F_{N \times(N-1)} & \varepsilon G_{N \times N}^{(1)} & \cdots & \varepsilon G_{N \times(N+1)}^{(j)} & \cdots & \varepsilon G_{N \times N}^{(m)} \\
\bar{G}_{N \times(N-1)}^{(1)} & \bar{F}_{N \times N} & \cdots & \mathbf{0} & \cdots & \mathbf{0} \\
\vdots & \vdots & \ddots & \vdots & \ddots & \vdots \\
\bar{G}_{N \times(N-1)}^{(j)} & \mathbf{0} & \cdots & \bar{F}_{N \times(N+1)} & \cdots & \mathbf{0} \\
\vdots & \vdots & \ddots & \vdots & \ddots & \vdots \\
\bar{G}_{N \times(N-1)}^{(m)} & \mathbf{0} & \cdots & \mathbf{0} & \cdots & \bar{F}_{N \times N}
\end{array}\right| \quad(1 \leq j \leq m),
$$

where $F_{N \times M}=A_{N \times N} \Theta_{N \times N}^{+} \Lambda_{N \times M}^{+}, G_{N \times M}^{(j)}=B_{N \times N}^{(j)} \Theta_{N \times N}^{-} \Lambda_{N \times M}^{-}(M=N-1, N, N+1)$. Via the multi-component Wronskian identity (32), we know that

$$
u=-2 \varepsilon \sum_{j=1}^{m}\left|p_{j}^{\prime}\right|^{2}=8 \sum_{n=1}^{m} \frac{\chi_{j}^{(1)} \chi_{j}^{(2)}}{\tau^{2}}=\frac{2\left(\tau \tau_{x x}-\tau_{x}^{2}\right)}{\tau^{2}}=2(\ln \tau)_{x x}
$$

which implies that $u$ is a real function.

Proposition 3.2. With $\Phi_{k}=\left(f_{k}, g_{k}^{(1)}, \ldots, g_{k}^{(m)}\right)^{\top}$ given by Eqs. (33a) and (33b) for $1 \leq k \leq K$, the KPI equation admits the solution $u=2(\ln \tau)_{x x}$, where the tau function $\tau$ is a reduced multi-component Wronskian given in Eq. (35). 


\section{Soliton solutions to the KP equation}

In this section, we explore the soliton solutions to the KPII equation generated by the unreduced multicomponent Wronskian (30), and to the KPI equation generated by the reduced multi-component Wronskian (35).

\subsection{Fully-resonant soliton solutions to the KPII equation}

As for the KPII equation, one might naturally ask whether the soliton solutions generated by the multicomponent Wronskian (30) are different from those by the single Wronskian (2). Since the multi-component Wronskian (30) with $m>1$ generates singular solutions for the generic choice of parameters, we in this subsection discuss the soliton solutions in $u=2(\ln \tau)_{x x}$ with

$$
\tau=\left|A_{2 N \times 2 N} \Theta_{2 N \times 2 N}^{+} \Lambda_{2 N \times N}^{+}, B_{2 N \times 2 N}^{(1)} \Theta_{2 N \times 2 N}^{-} \Lambda_{2 N \times N}^{-}\right| .
$$

For convenience, we take the coefficient matrix $B_{2 N \times 2 N}^{(1)}=B_{2 N \times 2 N}=\operatorname{diag}\left(-b_{1}, \ldots,-b_{2 N}\right)$ with $b_{k}=$ $b_{k}^{(1)}$ for $k \in[2 N]:=\{1,2, \ldots, 2 N\}$, and for comparison with the soliton solutions in Refs. [17-20] we assume that $\theta_{k}=-\kappa_{k} x-\sigma \kappa_{k}^{2} y-\kappa_{k}^{3} t$, where $\kappa_{k}=-2 \lambda_{k}$ and $\left\{\kappa_{k}\right\}_{k=1}^{2 N}$ are without loss of generality ordered as $\kappa_{1}<\kappa_{2} \cdots<\kappa_{2 N}$.

By using the Laplace expansion technique and Binet-Cauchy theorem, we can expand the function $\tau$ in Eq. (39) as follows:

$$
\tau=\sum_{\substack{\mathcal{I}_{N} \cap \mathcal{J}_{N}=\varnothing, \mathcal{I}_{N} \cup \mathcal{J}_{N}=[2 N]}}(-1)^{N+\sum_{n=1}^{N} i_{n}} \prod_{n=1}^{N} a_{i_{n}} b_{j_{n}} \prod_{1 \leq n<l \leq N}\left(\frac{1}{2} \kappa_{i_{n}}-\frac{1}{2} \kappa_{i_{l}}\right)\left(\frac{1}{2} \kappa_{j_{n}}-\frac{1}{2} \kappa_{j_{l}}\right) \exp \left[\frac{1}{2} \sum_{n=1}^{N}\left(\theta_{i_{n}}-\theta_{j_{n}}\right)\right],
$$

where $\mathcal{I}_{N}=\left\{i_{1}, \ldots, i_{N}\right\}$ and $\mathcal{J}_{N}=\left\{j_{1}, \ldots, j_{N}\right\}$ are two subsets of $[2 N]$ with $1 \leq i_{1}<\cdots<i_{N} \leq 2 N$ and $1 \leq j_{1}<\cdots<j_{N} \leq 2 N$. In order to ensure that the solution $u$ to the KPII equation resulting from the function $\tau$ in Eq. (39) is non-trivial and non-singular, the coefficients $a_{1}, \ldots, a_{2 N}, b_{1}, \ldots, b_{2 N}$ are required to satisfy the following three conditions:

(i) $a_{k}$ and $b_{k}$ are not equal to zero for the same $k \in[2 N]$;

(ii) $\left|\left\{a_{k} \mid a_{k} \neq 0\right\}\right|=N+L$ and $\left|\left\{b_{k} \mid b_{k} \neq 0\right\}\right|=N+M$, where $1 \leq L, M \leq N$ and $|\cdot|$ denotes the number of elements in a set;

(iii) $a_{k} a_{k+1} b_{k} b_{k+1} \leq 0$ for $1 \leq k \leq 2 N-1$.

Here, condition (i) requires that the function $\tau$ does not reduce to zero; condition (ii) requires that the function $\tau$ contains at least two exponentials; condition (iii) requires that the function $\tau$ is sign definite, so that $\tau$ has no zeros for all $(x, y, t) \in \mathbb{R}^{3}$. 
Considering that there might be some zeros among the coefficients $a_{1}, \ldots, a_{2 N}, b_{1}, \ldots, b_{2 N}$, we assume that $a_{i_{n}^{*}}=0$ for $n \in\left[L^{\prime}\right]$ and $b_{j_{n}^{*}}=0$ for $n \in\left[M^{\prime}\right]$, where $L^{\prime}=N-L, M^{\prime}=N-M, 1 \leq i_{1}^{*}<\cdots<$ $i_{L^{\prime}}^{*} \leq 2 N, 1 \leq j_{1}^{*}<\cdots<j_{M^{\prime}}^{*} \leq 2 N$ and $i_{n}^{*} \neq j_{l}^{*}$. Thus, the function $\tau$ in Eq. (40) can be expressed as

$$
\tau=\sum_{\substack{\mathcal{I}_{M} \cap \mathcal{J}_{L}=\varnothing, \mathcal{I}_{M} \cap \mathcal{J}_{M^{\prime}}^{*}=\varnothing, \mathcal{J}_{L} \cap \mathcal{I}_{L^{\prime}}^{*}=\varnothing \\ \mathcal{I}_{M} \cup \mathcal{J}_{L}=[2 N]\left(\mathcal{I}_{L^{\prime}}^{*} \cup \mathcal{J}_{M^{\prime}}^{*}\right)}}(-1)^{N+\sum_{n=1}^{M} i_{n}+\sum_{n=1}^{M^{\prime}} j_{n}^{*}} \prod_{n=1}^{L^{\prime}} \Upsilon_{i_{n}^{*}} \prod_{n=1}^{M^{\prime}} \Upsilon_{j_{n}^{*}} \prod_{n=1}^{M} a_{i_{n}} \prod_{n=1}^{L} b_{j_{n}}
$$

$$
\times \prod_{1 \leq n<l \leq M}\left(\frac{1}{2} \kappa_{i_{n}}-\frac{1}{2} \kappa_{i_{l}}\right) \prod_{1 \leq n<l \leq L}\left(\frac{1}{2} \kappa_{j_{n}}-\frac{1}{2} \kappa_{j_{l}}\right) \exp \left[\frac{1}{2}\left(\sum_{n=1}^{M} \theta_{i_{n}}+\sum_{n=1}^{M^{\prime}} \theta_{j_{n}^{*}}-\sum_{n=1}^{L} \theta_{j_{n}}-\sum_{n=1}^{L^{\prime}} \theta_{i_{n}^{*}}\right)\right],
$$

where $\mathcal{I}_{M}=\left\{i_{1}, \ldots, i_{M}\right\}, \mathcal{J}_{L}=\left\{j_{1}, \ldots, j_{L}\right\}, \mathcal{I}_{L^{\prime}}^{*}=\left\{i_{1}^{*}, \ldots, i_{L^{\prime}}^{*}\right\}, \mathcal{J}_{M^{\prime}}^{*}=\left\{j_{1}^{*}, \ldots, j_{M^{\prime}}^{*}\right\}, \Upsilon_{i_{n}^{*}}$ and $\Upsilon_{j_{n}^{*}}$ are given by

$$
\begin{aligned}
& \Upsilon_{i_{n}^{*}}=b_{i_{n}^{*}} \prod_{j_{l}<i_{n}^{*}}\left(\frac{1}{2} \kappa_{j_{l}}-\frac{1}{2} \kappa_{i_{n}^{*}}\right) \prod_{j_{l}>i_{n}^{*}}\left(\frac{1}{2} \kappa_{i_{n}^{*}}-\frac{1}{2} \kappa_{j_{l}}\right) \quad\left(1 \leq n \leq L^{\prime}\right), \\
& \Upsilon_{j_{n}^{*}}=a_{j_{n}^{*}} \prod_{i_{l}<j_{n}^{*}}\left(\frac{1}{2} \kappa_{i_{l}}-\frac{1}{2} \kappa_{j_{n}^{*}}\right) \prod_{i_{l}>j_{n}^{*}}\left(\frac{1}{2} \kappa_{j_{n}^{*}}-\frac{1}{2} \kappa_{i_{l}}\right) \quad\left(1 \leq n \leq M^{\prime}\right) .
\end{aligned}
$$

With scaling transformation, the function $\tau$ in Eq. (41) is further equivalent to

$$
\begin{aligned}
& \tau^{\prime}=\tau /\left\{\sum_{\substack{\mathcal{I}_{M} \cap \mathcal{J}_{L}=\varnothing, \mathcal{I}_{M} \cap \mathcal{J}_{M}^{*}=\varnothing, \mathcal{J}_{L} \cap \mathcal{I}_{L^{\prime}}^{*}=\varnothing \\
\mathcal{I}_{M} \cup \mathcal{J}_{L}=[2 N] \backslash\left(\mathcal{I}_{L^{\prime}}^{*} \cup \mathcal{J}_{M^{\prime}}^{*}\right)}}(-1)^{L^{\prime}+\sum_{n=1}^{M^{\prime}} j_{n}^{*}} \prod_{n=1}^{L^{\prime}} \Upsilon_{i_{n}^{*}} \prod_{n=1}^{M^{\prime}} \Upsilon_{j_{n}^{*}} \prod_{n=1}^{M} a_{i_{n}} \prod_{n=1}^{L} a_{j_{n}}\right. \\
& \left.\times\left(\frac{1}{2}\right)^{\frac{M(M+1)}{2}+\frac{L(L+1)}{2}} \exp \left[\frac{1}{2}\left(\sum_{n=1}^{M^{\prime}} \theta_{j_{n}^{*}}-\sum_{n=1}^{M} \theta_{i_{n}}-\sum_{n=1}^{L} \theta_{j_{n}}-\sum_{n=1}^{L^{\prime}} \theta_{i_{n}^{*}}\right)\right]\right\} \\
& =\sum_{\mathcal{I}_{M} \cap \mathcal{J}_{L}=\varnothing, \mathcal{I}_{M} \cap \mathcal{J}_{M^{\prime}}^{*}=\varnothing, \mathcal{J}_{L} \cap \mathcal{I}_{L^{\prime}}^{*}=\varnothing}(-1)^{L+\sum_{n=1}^{M} i_{n}} \prod_{n=1}^{L} b_{j_{n}}^{\prime} \\
& \mathcal{I}_{M} \cup \mathcal{J}_{L}=[2 N] \backslash\left(\mathcal{I}_{L^{\prime}}^{*} \cup \mathcal{J}_{M^{\prime}}^{*}\right) \\
& \times \prod_{1 \leq n<l \leq M}\left(\kappa_{i_{n}}-\kappa_{i_{l}}\right) \prod_{1 \leq n<l \leq L}\left(\kappa_{j_{n}}-\kappa_{j_{l}}\right) \exp \left(\sum_{n=1}^{M} \theta_{i_{n}}\right) \quad\left(b_{j_{n}}^{\prime}=b_{j_{n}} / a_{j_{n}}\right),
\end{aligned}
$$

in generating the solution $u$ of the KPII equation. Without loss of generality, we suppose that $\mathcal{I}_{M} \cup \mathcal{J}_{L}=$ $[M+L]$ and $\mathcal{I}_{L^{\prime}}^{*} \cup \mathcal{J}_{M^{\prime}}^{*}=\{M+L+1, \ldots, 2 N\}$. We notice that the function $\tau^{\prime}$ in Eq. (43) contains all possible combinations of $M$ phases out of $\left\{\theta_{1}, \ldots, \theta_{M+L}\right\}$, which is the same as the case of a family of fully-resonant soliton solutions to the KPII equation discussed in Ref. [16]. Therefore, we immediately come to the following results:

Proposition 4.1. The dominant exponentials of the function $\tau^{\prime}$ in adjacent regions of the xy-plane as $\sigma y \rightarrow$ $\pm \infty$ contain $M-1$ common phases and differ by only one phase, and the transition between two such 
exponentials occurs along the line defined by $L_{i j}: \theta_{i}=\theta_{j}$ where $j=i+M$ for $i \in[L]$ as $\sigma y \rightarrow \infty$ and $j=i+L$ for $i \in[M]$ as $\sigma y \rightarrow-\infty$. In a neighborhood of the transition line $L_{i j}$ as $\sigma y \rightarrow \pm \infty$, the asymptotic behavior of $u=2\left(\ln \tau^{\prime}\right)_{x x}$ is determined by

$$
u \sim u_{[i, j]}^{ \pm}=2 \frac{\partial^{2}}{\partial x^{2}} \ln \left(C_{i}^{ \pm} \mathrm{e}^{\theta_{i}}+C_{j}^{ \pm} \mathrm{e}^{\theta_{j}}\right)=A_{[i, j]} \operatorname{sech}^{2}\left(\mathbf{K}_{[i, j]} \cdot \mathbf{r}+\Omega_{[i, j]} t+\ln \frac{C_{j}^{ \pm}}{C_{i}^{ \pm}}\right),
$$

with

$$
\begin{aligned}
& C_{i}^{+}=(-1)^{i} b_{j}^{\prime} \prod_{i+1 \leq n \leq j-1}\left(\kappa_{i}-\kappa_{n}\right) \prod_{1 \leq n \leq i-1}\left(\kappa_{n}-\kappa_{j}\right) \prod_{j+1 \leq n \leq M+L}\left(\kappa_{j}-\kappa_{n}\right), \\
& C_{j}^{+}=(-1)^{j} b_{i}^{\prime} \prod_{i+1 \leq n \leq j-1}\left(\kappa_{n}-\kappa_{j}\right) \prod_{1 \leq n \leq i-1}\left(\kappa_{n}-\kappa_{i}\right) \prod_{j+1 \leq n \leq M+L}\left(\kappa_{i}-\kappa_{n}\right), \\
& C_{i}^{-}=(-1)^{i} b_{j}^{\prime} \prod_{1 \leq n \leq i-1}\left(\kappa_{n}-\kappa_{i}\right) \prod_{j+1 \leq n \leq M+L}\left(\kappa_{i}-\kappa_{n}\right) \prod_{i+1 \leq n \leq j-1}\left(\kappa_{n}-\kappa_{j}\right), \\
& C_{j}^{-}=(-1)^{j} b_{i}^{\prime} \prod_{1 \leq n \leq i-1}\left(\kappa_{n}-\kappa_{j}\right) \prod_{j+1 \leq n \leq M+L}\left(\kappa_{j}-\kappa_{n}\right) \prod_{i+1 \leq n \leq j-1}\left(\kappa_{i}-\kappa_{n}\right), \\
& \mathbf{r}=(x, y), \quad \mathbf{K}_{[i, j]}=\left[\frac{1}{2}\left(\kappa_{j}-\kappa_{i}\right), \frac{1}{2 \sigma}\left(\kappa_{j}^{2}-\kappa_{i}^{2}\right)\right], \\
& A_{[i, j]}=\frac{1}{2}\left(\kappa_{j}-\kappa_{i}\right)^{2}, \quad \Omega_{[i, j]}=\frac{1}{2}\left(\kappa_{j}^{3}-\kappa_{i}^{3}\right),
\end{aligned}
$$

where $u_{[i, j]}^{ \pm}$defines the asymptotic line soliton $[i, j]$ as $\sigma y \rightarrow \pm \infty$, and $A_{[i, j]}, \mathbf{K}_{[i, j]}$ and $\Omega_{[i, j]}$ respectively represent the amplitude, wave vector and frequency of asymptotic soliton.

We remark that the function $\tau^{\prime}$ in Eq. (43) generates two families of fully-resonant soliton solutions: one is $(L, M)$-soliton configuration with $L$ asymptotic solitons as $y \rightarrow \infty$ and $M$ asymptotic solitons as $y \rightarrow-\infty$ for $\sigma=1$ (see Figure 1), and the other is $(M, L)$-soliton configuration with $M$ asymptotic solitons as $y \rightarrow \infty$ and $L$ asymptotic solitons as $y \rightarrow-\infty$ for $\sigma=-1$ (see Figure 2). Such two families of solutions correspond to two special cases of the soliton solutions generated by the function $\tau$ in Eq. (2) because the soliton amplitude $A_{[i, j]}$, wave vector $\mathbf{K}_{[i, j]}$ and frequency $\Omega_{[i, j]}$ in the asymptotic expression (44) are the same as those given in Refs. [17, 19, 20].

\subsection{Ordinary $N$-soliton solution to the KPI equation}

In this subsection, we are devoted to discussing the soliton solutions to the KPI equation generated by the function $\tau$ in Eq. (35) with $m=1$ and $\varepsilon=-1$, i.e.,

$$
\tau=\left|\begin{array}{cc}
F_{N \times N} & -G_{N \times N}^{(1)} \\
\bar{G}_{N \times N}^{(1)} & \bar{F}_{N \times N}
\end{array}\right|
$$


where $\tau$ is a real-valued function and has no zeros for all $(x, y, t) \in \mathbb{R}^{3}$. Our consideration is based on the following two facts: (a) The solutions generated by the $(m+1)$-component Wronskian (35) do not contain more valid free parameters than those by the two-component Wronskian (45); (b) The function $\tau$ in Eq. (35) with $\varepsilon=1$ has zeros for some $(x, y, t) \in \mathbb{R}^{3}$. For convenience, we define that $b_{k}=b_{k}^{(1)}$ and $\lambda_{k}=\frac{1}{4}\left(\mu_{k}+\sigma \nu_{k}\right)$ for $k \in[N]$, where $\sigma= \pm \mathrm{i}, \mu_{k}$ and $\nu_{k}$ are real constants. Without loss of generality, we assume that $a_{k}=1, b_{k} \neq 0$ for $k \in[N]$ and $\left\{\nu_{k}\right\}_{k=1}^{N}$ are well ordered as $\nu_{1}<\nu_{2}<\cdots<\nu_{N}$.

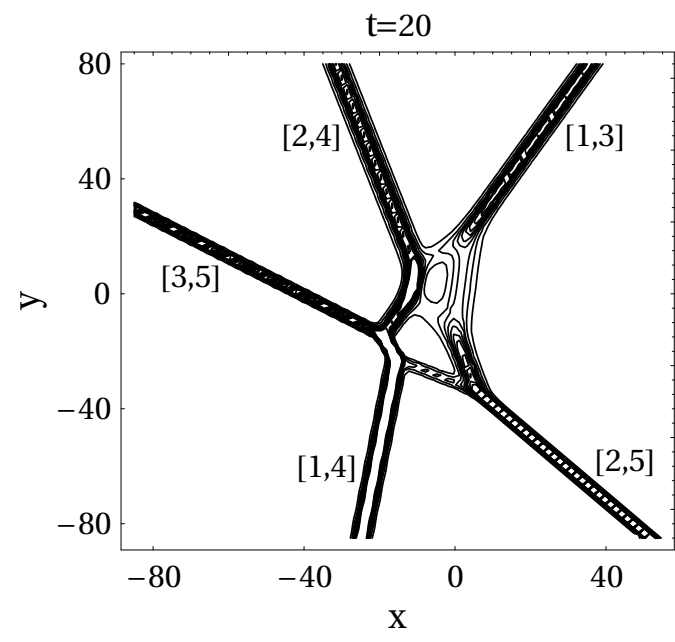

Figure 1. A fully-resonant $(3,2)$ soliton solution to the KPII equation generated by the function $\tau^{\prime}$ in Eq. (43) with the parameters chosen as $L=3, M=2, \sigma=1$, $b_{1}^{\prime}=b_{2}^{\prime}=b_{3}^{\prime}=b_{4}^{\prime}=b_{5}^{\prime}=1$, $\kappa_{1}=-0.8, \kappa_{2}=-0.35, \kappa_{3}=$ $0.25, \kappa_{4}=0.65$ and $\kappa_{5}=1.25$.

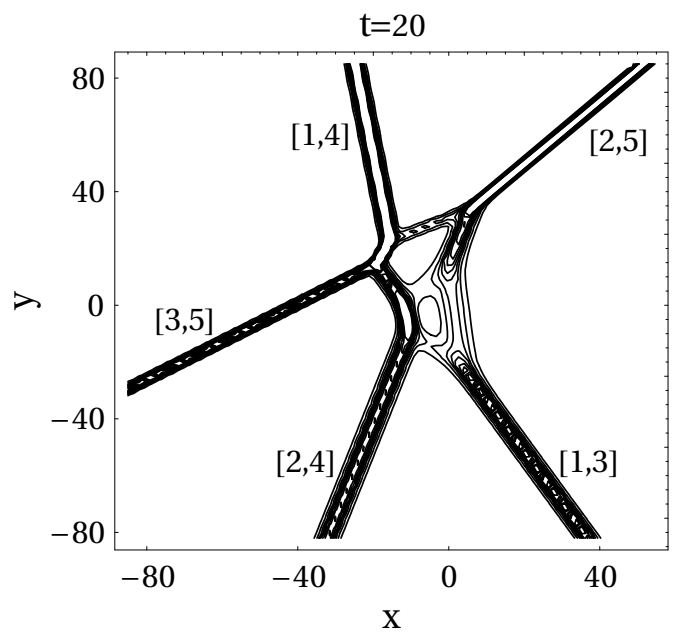

Figure 2. A fully-resonant $(2,3)$ soliton solution to the KPII equation generated by the function $\tau^{\prime}$ in Eq. (43) with the same parameters as those in Figure 1 except for $\sigma=-1$.

For the case $N=1$, the solution $u=2(\ln \tau)_{x x}$ with $\tau$ given by Eq. (45) can be expressed as

$$
u=\frac{1}{2} \mu_{1}^{2} \operatorname{sech}^{2}\left[\frac{1}{2} \mu_{1} x-\frac{1}{2} \mu_{1} \nu_{1} y+\frac{1}{8} \mu_{1}\left(\mu_{1}^{2}-3 \nu_{1}^{2}\right) t-\ln \left|b_{1}\right|\right],
$$

which describes the one-soliton solution to the KPI equation (see Figure 3), where the amplitude, wave vector and frequency of soliton are respectively given by $\frac{1}{2} \mu_{1}^{2},\left(\frac{1}{2} \mu_{1},-\frac{1}{2} \mu_{1} \nu_{1}\right)$ and $\frac{1}{8} \mu_{1}\left(\mu_{1}^{2}-3 \nu_{1}^{2}\right)$. It is mentioned that such line-soliton solution is in accordance with those obtained by the Hirota method [6] and inverse scattering transform [37].

For the case $N \geq 2$, we can employ the results of Ref. [29] to analyze the asymptotic behavior of the function $\tau$ in Eq. (45). According to Lemma 4.1 in Ref. [29], we know that the function $\tau$ in Eq. (45) can be 
expanded as the sum of such exponential terms as $\exp \left[\frac{1}{2} \sum_{k=1}^{N}\left(r_{k} \theta_{k}+s_{k} \bar{\theta}_{k}\right)\right]$, where $r_{k}, s_{k} \in\{-1,1\}$, $\left|\left\{r_{k} \mid r_{k}=-1\right\}\right|=\left|\left\{s_{k} \mid s_{k}=-1\right\}\right|$ and $\left|\left\{r_{k} \mid r_{k}=1\right\}\right|=\left|\left\{s_{k} \mid s_{k}=1\right\}\right|$. Further using Theorem 4.4 in Ref. [29], we obtain that along the line $\theta_{n}+\bar{\theta}_{n}=0(1 \leq n \leq N)$ as $y \rightarrow \pm \infty$ for finite values of $t$, the solution $u=2(\ln \tau)_{x x}$ is asymptotically determined by

$$
\begin{aligned}
u \sim u_{[n, \bar{n}]}^{ \pm}=2 \frac{\partial^{2}}{\partial x^{2}} \ln \left(\zeta_{n}^{ \pm} \exp \left\{\frac{1}{2}\left[\theta_{n}+\bar{\theta}_{n}+\sum_{k \neq n} \epsilon_{k n}^{ \pm}\left(\theta_{k}+\bar{\theta}_{k}\right)\right]\right\}\right. \\
\left.+\eta_{n}^{ \pm} \exp \left\{\frac{1}{2}\left[-\theta_{n}-\bar{\theta}_{n}+\sum_{k \neq n} \epsilon_{k n}^{ \pm}\left(\theta_{k}+\bar{\theta}_{k}\right)\right]\right\}\right) \\
=\frac{1}{2} \mu_{n}^{2} \operatorname{sech}^{2}\left(\frac{\theta_{n}+\bar{\theta}_{n}}{2}+\ln \sqrt{\zeta_{n}^{ \pm} / \eta_{n}^{ \pm}}\right) \quad(1 \leq n \leq N),
\end{aligned}
$$

where $u_{[n, \bar{n}]}^{ \pm}$defines the $n$-th asymptotic line soliton $[n, \bar{n}]$ as $y \rightarrow \pm \infty, \zeta_{n}^{ \pm}$and $\eta_{n}^{ \pm}$correspond to the coefficients of two dominant exponentials in the expansion of $\tau$, the parameters $\epsilon_{k n}^{ \pm}$'s are defined as

$$
\epsilon_{k n}^{-}=\left\{\begin{array}{cl}
-1, & \text { for } k \in \mathcal{B}_{n}^{(\mathrm{I})} \cup \mathcal{B}_{n}^{(\mathrm{II})}, \\
1, & \text { for } k \in \mathcal{B}_{n}^{(\mathrm{III})} \cup \mathcal{B}_{n}^{(\mathrm{IV})},
\end{array} \quad \epsilon_{k n}^{+}=\left\{\begin{array}{cl}
1, & \text { for } k \in \mathcal{B}_{n}^{(\mathrm{I})} \cup \mathcal{B}_{n}^{(\mathrm{II})}, \\
-1, & \text { for } k \in \mathcal{B}_{n}^{(\mathrm{III})} \cup \mathcal{B}_{n}^{(\mathrm{IV})},
\end{array}\right.\right.
$$

with $\mathcal{B}_{n}^{(\mathrm{I})}=\left\{l \mid \mu_{l}>0, l=1, \ldots, n-1\right\}, \mathcal{B}_{n}^{(\mathrm{II})}=\left\{l \mid \mu_{l}<0, l=n+1, \ldots, N\right\}, \mathcal{B}_{n}^{(\mathrm{III})}=\left\{l \mid \mu_{l}<0, l=\right.$ $1, \ldots, n-1\}$ and $\mathcal{B}_{n}^{(\mathrm{IV})}=\left\{l \mid \mu_{l}>0, l=n+1, \ldots, N\right\}$.

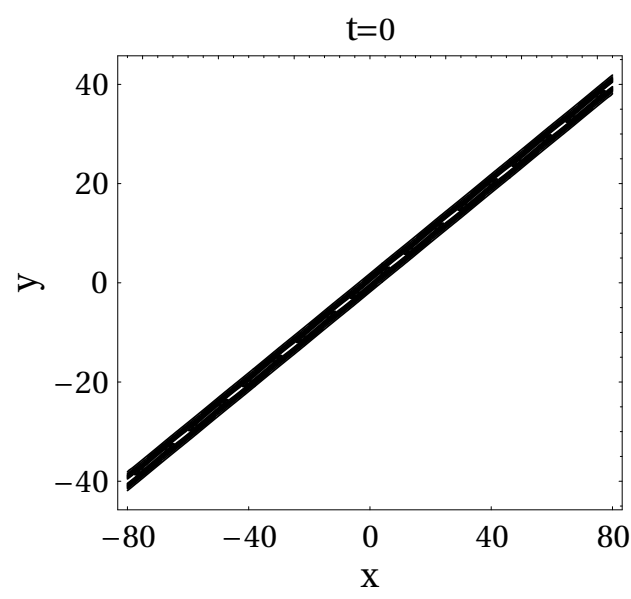

Figure 3. A one-soliton solution to the KPI equation generated by the function $\tau$ in Eq. (45) with the parameters chosen as $N=1, \sigma=$ i, $\mu_{1}=1, \nu_{1}=2$ and $b_{1}=1$.

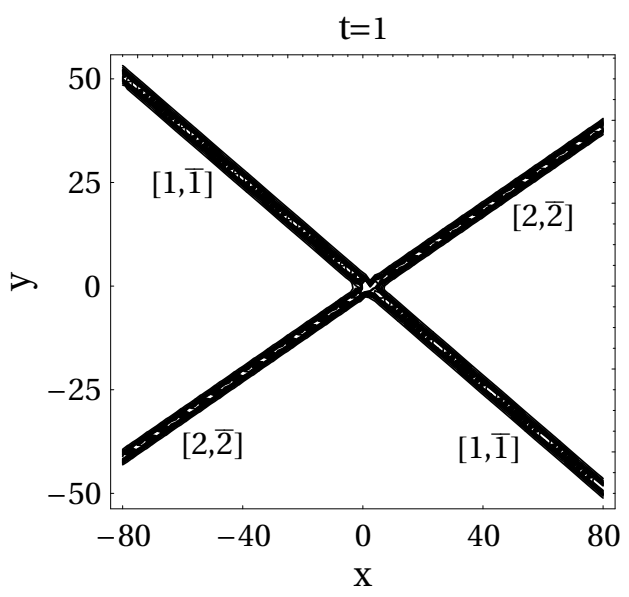

Figure 4. An obliquely-colliding two-soliton solution to the KPI equation generated by the function $\tau$ in Eq. (45) with the parameters chosen as $N=2, \sigma=\mathrm{i}, \mu_{1}=$ $\mu_{2}=1, \nu_{1}=-1.6, \nu_{2}=2$ and $b_{1}=b_{2}=1$. 
The asymptotic expression (47) shows that the $n$-th asymptotic soliton as $y \rightarrow \infty$ has the same velocity and amplitude as the $n$-th asymptotic soliton as $y \rightarrow-\infty$ except for a slight phase shift, as displayed in Figure 4. Therefore, the $N$-soliton solution generated by the function $\tau$ in Eq. (45) reflect only the ordinary elastic collisions of line solitons in the KPI equation. Particularly with $\nu_{n}=\nu_{k}(n \neq k)$, the $n$-th and $k$-th asymptotic solitons propagate parallel to each other (see Figure 5), but they exhibit the bound state [38] at the moment of collision (see Figure 6). It is an interesting question whether the KPI equation also admits the inelastic soliton collisions (in which the numbers, amplitudes or directions of asymptotic solitons are not the same as $y \rightarrow \pm \infty$ ), like the cases in the KPII equation.

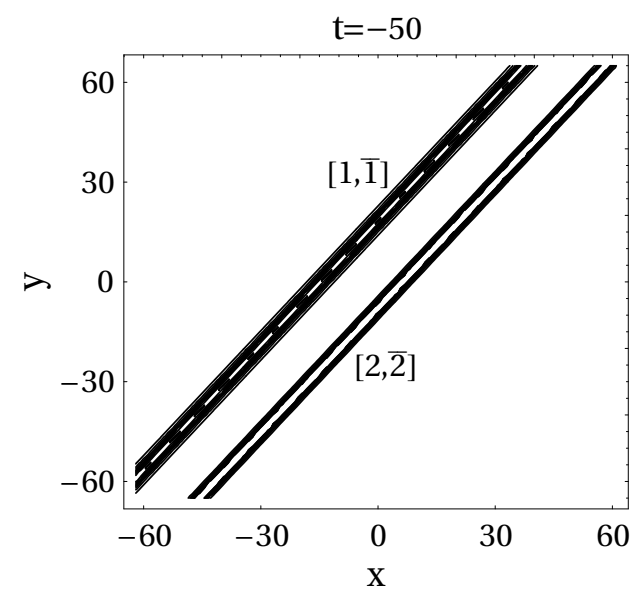

Figure 5. Parallel propagation of two line solitons generated by the function $\tau$ in Eq. (45) with the parameters chosen as $N=2, \sigma=\mathrm{i}$, $\mu_{1}=1, \mu_{2}=1.5, \nu_{1}=\nu_{2}=0.8$ and $b_{1}=b_{2}=1$.

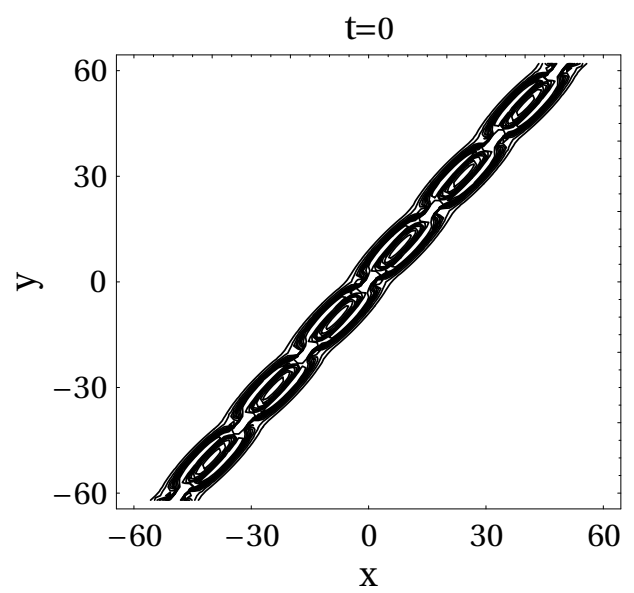

Figure 6. Bound state between two line solitons at the moment of collision generated by the function $\tau$ in Eq. (45) with the same parameters as those in Figure 5.

\section{Conclusions}

In this paper, we have constructed the $N$-th iterated DT for the second- and third-order $m$-coupled AKNS systems (3) and (4), and rigorously proved the form-invariance of their Lax representation (6). Then, using Cramer's rule and based on the integrable decomposition from the KP equation (1) to Systems (3) and (4), we have derived the unreduced multi-component Wronskian solution to the KPII equation and a reduced multi-component Wronskian solution to the KPI equation, which implies that the multi-component Wronskian is a third form of the tau function of the KP equation in addition to the Wronskian and Grammian. Further, by analyzing algebraic properties of the unreduced and reduced two-component Wronskians, we 
have obtained two families of fully-resonant line-soliton solutions which, in general, contain unequal numbers of asymptotic solitons as $y \rightarrow \mp \infty$ to the KPII equation, and the ordinary $N$-soliton solution which generally describes the elastic collisions of line solitons to the KPI equation. In particular, we have found that the KPI line solitons which propagate in parallel can exhibit the bound state at the moment of collision. It is remarkable that the double Wronskian representation of the $N$-soliton solution to the KPI equation has been revealed in this work for the first time.

\section{Acknowledgements}

TX would like to thank the helpful discussions with Professor G. Biondini. This work has been supported by the Science Foundation of China University of Petroleum, Beijing (No. BJ-2011-04), and by the National Natural Science Foundations of China under Grants No. 11071257 and No. 11101421. 


\section{Appendix A Proof of Lemma 2.2}

Proof. First, we make use of Eqs. (15) to compute $T^{*}\left(\lambda_{k}\right)$ as

$$
T^{*}\left(\lambda_{k}\right)=\left(\begin{array}{cccc}
\Delta_{0}\left(\lambda_{k}\right) & -\Delta_{1}\left(\lambda_{k}\right) & \cdots & (-1)^{m} \Delta_{m}\left(\lambda_{k}\right) \\
\varpi_{k}^{(1)} \Delta_{0}\left(\lambda_{k}\right) & -\varpi_{k}^{(1)} \Delta_{1}\left(\lambda_{k}\right) & \cdots & (-1)^{m} \varpi_{k}^{(1)} \Delta_{m}\left(\lambda_{k}\right) \\
\vdots & \vdots & \ddots & \vdots \\
\varpi_{k}^{(m)} \Delta_{0}\left(\lambda_{k}\right) & -\varpi_{k}^{(m)} \Delta_{1}\left(\lambda_{k}\right) & \cdots & (-1)^{m} \varpi_{k}^{(m)} \Delta_{m}\left(\lambda_{k}\right)
\end{array}\right)
$$

with

$$
\Delta_{0}\left(\lambda_{k}\right)=\left|\begin{array}{ccc}
\delta_{11}\left(\lambda_{k}\right) & \cdots & \delta_{1 m}\left(\lambda_{k}\right) \\
\vdots & \ddots & \vdots \\
\delta_{m 1}\left(\lambda_{k}\right) & \cdots & \delta_{m m}\left(\lambda_{k}\right)
\end{array}\right|, \quad \Delta_{i}\left(\lambda_{k}\right)=\left|\begin{array}{ccc}
\beta_{1}\left(\lambda_{k}\right) & \cdots & \beta_{m}\left(\lambda_{k}\right) \\
\delta_{11}\left(\lambda_{k}\right) & \cdots & \delta_{1 m}\left(\lambda_{k}\right) \\
\vdots & \ddots & \vdots \\
\delta_{i-1,1}\left(\lambda_{k}\right) & \cdots & \delta_{i-1, m}\left(\lambda_{k}\right) \\
\delta_{i+1,1}\left(\lambda_{k}\right) & \cdots & \delta_{i+1, m}\left(\lambda_{k}\right) \\
\vdots & \ddots & \vdots \\
\delta_{m 1}\left(\lambda_{k}\right) & \cdots & \delta_{m m}\left(\lambda_{k}\right)
\end{array}\right| \quad(1 \leq i \leq m) .
$$

Then, we expand $\left[T_{x}\left(\lambda_{k}\right)+T\left(\lambda_{k}\right) U\left(\lambda_{k}\right)\right] T^{*}\left(\lambda_{k}\right),\left[T_{y}\left(\lambda_{k}\right)+T\left(\lambda_{k}\right) V\left(\lambda_{k}\right)\right] T^{*}\left(\lambda_{k}\right)$ and $\left[T_{t}\left(\lambda_{k}\right)+T\left(\lambda_{k}\right)\right.$ $\left.W\left(\lambda_{k}\right)\right] T^{*}\left(\lambda_{k}\right)$, obtaining that

$$
\begin{aligned}
& u_{1 l}\left(\lambda_{k}\right)=(-1)^{l-1} \Delta_{l-1}\left(\lambda_{k}\right)\left\{\alpha_{x}\left(\lambda_{k}\right)+\lambda_{k} \alpha\left(\lambda_{k}\right)+\sum_{j=1}^{m} \beta_{j}\left(\lambda_{k}\right) q_{j}\right. \\
& \left.+\sum_{j=1}^{m} \varpi_{k}^{(j)}\left[\beta_{j, x}\left(\lambda_{k}\right)+\alpha\left(\lambda_{k}\right) p_{j}-\lambda_{k} \beta_{j}\left(\lambda_{k}\right)\right]\right\} \quad(1 \leq l \leq m+1) \\
& u_{i+1, l}\left(\lambda_{k}\right)=(-1)^{l-1} \Delta_{l-1}\left(\lambda_{k}\right)\left\{\gamma_{i, x}\left(\lambda_{k}\right)+\lambda_{k} \gamma_{i}\left(\lambda_{k}\right)+\sum_{j=1}^{m} \delta_{i j}\left(\lambda_{k}\right) q_{j}\right. \\
& \left.+\sum_{j=1}^{m} \varpi_{k}^{(j)}\left[\delta_{i j, x}\left(\lambda_{k}\right)+\gamma_{i}\left(\lambda_{k}\right) p_{j}-\lambda_{k} \delta_{i j}\left(\lambda_{k}\right)\right]\right\} \quad(1 \leq i \leq m ; 1 \leq l \leq m+1), \\
& v_{1 l}\left(\lambda_{k}\right)=(-1)^{l-1} \Delta_{l-1}\left(\lambda_{k}\right)\left\{\alpha_{y}\left(\lambda_{k}\right)-\frac{1}{\sigma}\left[\alpha\left(\lambda_{k}\right)\left(2 \lambda_{k}^{2}-\sum_{n=1}^{m} p_{n} q_{n}\right)-\sum_{n=1}^{m} \beta_{n}\left(\lambda_{k}\right) q_{n, x}\right.\right. \\
& \left.\left.+2 \lambda_{k} \sum_{n=1}^{m} \beta_{n}\left(\lambda_{k}\right) q_{n}\right]\right\}+(-1)^{l-1} \Delta_{l-1}\left(\lambda_{k}\right) \sum_{j=1}^{m} \varpi_{k}^{(j)}\left\{\beta_{j, y}\left(\lambda_{k}\right)\right. \\
& \left.-\frac{1}{\sigma}\left[\alpha\left(\lambda_{k}\right)\left(2 \lambda_{k} p_{j}+p_{j, x}\right)-2 \lambda_{k}^{2} \beta_{j}\left(\lambda_{k}\right)+\sum_{n=1}^{m} \beta_{n}\left(\lambda_{k}\right) q_{n} p_{j}\right]\right\} \quad(1 \leq l \leq m+1), \\
& v_{i+1, l}\left(\lambda_{k}\right)=(-1)^{l-1} \Delta_{l-1}\left(\lambda_{k}\right)\left\{\gamma_{i, y}\left(\lambda_{k}\right)-\frac{1}{\sigma}\left[\gamma_{i}\left(\lambda_{k}\right)\left(2 \lambda_{k}^{2}-\sum_{n=1}^{m} p_{n} q_{n}\right)-\sum_{n=1}^{m} \delta_{i n}\left(\lambda_{k}\right) q_{n, x}\right.\right.
\end{aligned}
$$




$$
\left.\left.+2 \lambda_{k} \sum_{n=1}^{m} \delta_{i n}\left(\lambda_{k}\right) q_{n}\right]\right\}+(-1)^{l-1} \Delta_{l-1}\left(\lambda_{k}\right) \sum_{j=1}^{m} \varpi_{k}^{(j)}\left\{\delta_{i j, y}\left(\lambda_{k}\right)-\frac{1}{\sigma}\left[-2 \lambda_{k}^{2} \delta_{i j}\left(\lambda_{k}\right)\right.\right.
$$

A.5) $\left.\left.\quad+\gamma_{i}\left(\lambda_{k}\right)\left(2 \lambda_{k} p_{j}+p_{j, x}\right)+\sum_{n=1}^{m} \delta_{i n}\left(\lambda_{k}\right) q_{n} p_{j}\right]\right\} \quad(1 \leq i \leq m ; 1 \leq l \leq m+1)$

$$
\begin{aligned}
w_{1 l}\left(\lambda_{k}\right)= & (-1)^{l-1} \Delta_{l-1}\left(\lambda_{k}\right)\left\{\alpha_{t}\left(\lambda_{k}\right)+\left[4 \lambda_{k}^{3} \alpha\left(\lambda_{k}\right)-\alpha\left(\lambda_{k}\right) \sum_{n=1}^{m}\left(2 \lambda_{k} p_{n} q_{n}+p_{n, x} q_{n}-p_{n} q_{n, x}\right)\right.\right. \\
& \left.\left.+\sum_{n=1}^{m} \beta_{n}\left(\lambda_{k}\right)\left(4 \lambda_{k}^{2} q_{n}-2 \lambda_{k} q_{n, x}-2 q_{n} \sum_{r=1}^{m} p_{r} q_{r}+q_{n, x x}\right)\right]\right\}+(-1)^{l-1} \Delta_{l-1}\left(\lambda_{k}\right) \\
& \times \sum_{j=1}^{m} \varpi_{k}^{(j)}\left\{\beta_{j, t}\left(\lambda_{k}\right)+\left[\alpha\left(\lambda_{k}\right)\left(4 \lambda_{k}^{2} p_{j}+2 \lambda_{k} p_{j, x}-2 p_{j} \sum_{n=1}^{m} p_{n} q_{n}+p_{j, x x}\right)\right.\right.
\end{aligned}
$$

$$
\left.\left.-4 \lambda_{k}^{3} \beta_{j}\left(\lambda_{k}\right)+\left(2 \lambda_{k} p_{j}+p_{j, x}\right) \sum_{n=1}^{m} \beta_{n}\left(\lambda_{k}\right) q_{n}-p_{j} \sum_{n=1}^{m} \beta_{n}\left(\lambda_{k}\right) q_{n, x}\right]\right\}(1 \leq l \leq m+1),
$$

$$
w_{i+1, l}\left(\lambda_{k}\right)=(-1)^{l-1} \Delta_{l-1}\left(\lambda_{k}\right)\left\{\gamma_{i, t}\left(\lambda_{k}\right)+\left[4 \lambda_{k}^{3} \gamma_{i}\left(\lambda_{k}\right)-\gamma_{i}\left(\lambda_{k}\right) \sum_{n=1}^{m}\left(2 \lambda_{k} p_{n} q_{n}+p_{n, x} q_{n}-p_{n} q_{n, x}\right)\right.\right.
$$

$$
\begin{aligned}
& \left.\left.+\sum_{n=1}^{m} \delta_{i n}\left(\lambda_{k}\right)\left(4 \lambda_{k}^{2} q_{n}-2 \lambda_{k} q_{n, x}-2 q_{n} \sum_{r=1}^{m} p_{r} q_{r}+q_{n, x x}\right)\right]\right\}+(-1)^{l-1} \Delta_{l-1}\left(\lambda_{k}\right) \\
& \times \sum_{j=1}^{m} \varpi_{k}^{(j)}\left\{\delta_{i j, t}\left(\lambda_{k}\right)+\left[\gamma_{i}\left(\lambda_{k}\right)\left(4 \lambda_{k}^{2} p_{j}+2 \lambda_{k} p_{j, x}-2 p_{j} \sum_{n=1}^{m} p_{n} q_{n}+p_{j, x x}\right)-4 \lambda_{k}^{3} \delta_{i j}\left(\lambda_{k}\right)\right.\right.
\end{aligned}
$$

$$
\left.\left.+\left(2 \lambda_{k} p_{j}+p_{j, x}\right) \sum_{n=1}^{m} \delta_{i n}\left(\lambda_{k}\right) q_{n}-p_{j} \sum_{n=1}^{m} \delta_{i n}\left(\lambda_{k}\right) q_{n, x}\right]\right\} \quad(1 \leq i \leq m ; 1 \leq l \leq m+1) .
$$

Using Eqs. (15) and recalling that $\Phi_{k}=\left(f_{k}, g_{k}^{(1)}, \ldots, g_{k}^{(m)}\right)^{\top}$ satisfies System (6) with $\lambda=\lambda_{k}$, we obtain the derivatives of $\alpha\left(\lambda_{k}\right)$ and $\gamma_{i}\left(\lambda_{k}\right)$ with respect to $x, y$ and $t$ as follows:

$$
\begin{aligned}
& (\operatorname{Aas})\left(\lambda_{k}\right)=-\sum_{j=1}^{m}\left(q_{j}-2 \lambda_{k} \varpi_{k}^{(j)}-\varpi_{k}^{(j)} \sum_{n=1}^{m} p_{n} \varpi_{k}^{(n)}\right) \beta_{j}\left(\lambda_{k}\right)-\sum_{j=1}^{m} \varpi_{k}^{(j)} \beta_{j, x}\left(\lambda_{k}\right), \\
& \left(A_{\gamma, 9, x}\left(\lambda_{k}\right)=-\sum_{j=1}^{m}\left(q_{j}-2 \lambda_{k} \varpi_{k}^{(j)}-\varpi_{k}^{(j)} \sum_{n=1}^{m} p_{n} \varpi_{k}^{(n)}\right) \delta_{i j}\left(\lambda_{k}\right)-\sum_{j=1}^{m} \varpi_{k}^{(j)} \delta_{i j, x}\left(\lambda_{k}\right) \quad(1 \leq i \leq m),\right. \\
& \alpha_{y}\left(\lambda_{k}\right)=\frac{1}{\sigma} \sum_{j=1}^{m}\left(2 \lambda_{k} q_{j}-q_{j, x}-4 \lambda_{k}^{2} \varpi_{k}^{(j)}+q_{j} \sum_{n=1}^{m} p_{n} \varpi_{k}^{(n)}+\varpi_{k}^{(j)} \sum_{n=1}^{m} p_{n} q_{n}\right. \\
& \left.-2 \lambda_{k} \varpi_{k}^{(j)} \sum_{n=1}^{m} p_{n} \varpi_{k}^{(n)}-\varpi_{k}^{(j)} \sum_{n=1}^{m} p_{n, x} \varpi_{k}^{(n)}\right) \beta_{j}\left(\lambda_{k}\right)-\sum_{j=1}^{m} \varpi_{k}^{(j)} \beta_{j, y}\left(\lambda_{k}\right), \\
& \gamma_{i, y}\left(\lambda_{k}\right)=\frac{1}{\sigma} \sum_{j=1}^{m}\left(2 \lambda_{k} q_{j}-q_{j, x}-4 \lambda_{k}^{2} \varpi_{k}^{(j)}+q_{j} \sum_{n=1}^{m} p_{n} \varpi_{k}^{(n)}+\varpi_{k}^{(j)} \sum_{n=1}^{m} p_{n} q_{n}\right.
\end{aligned}
$$




$$
\left.-2 \lambda_{k} \varpi_{k}^{(j)} \sum_{n=1}^{m} p_{n} \varpi_{k}^{(n)}-\varpi_{k}^{(j)} \sum_{n=1}^{m} p_{n, x} \varpi_{k}^{(n)}\right) \delta_{i j}\left(\lambda_{k}\right)-\sum_{j=1}^{m} \varpi_{k}^{(j)} \delta_{i j, y}\left(\lambda_{k}\right)(1 \leq i \leq m),
$$

$$
\alpha_{t}\left(\lambda_{k}\right)=-\sum_{j=1}^{m}\left\{4 \lambda_{k}^{2} q_{j}-2 \lambda_{k} q_{j, x}-2 q_{j} \sum_{n=1}^{m} p_{n} q_{n}+q_{j, x x}-4 \lambda_{k}^{3} \varpi_{k}^{(j)}+2 \lambda_{k} q_{j} \sum_{n=1}^{m} p_{n} \varpi_{k}^{(n)}\right.
$$

$$
-q_{j, x} \sum_{n=1}^{m} p_{n} \varpi_{k}^{(n)}+q_{j} \sum_{n=1}^{m} p_{n, x} \varpi_{k}^{(n)}-\varpi_{k}^{(j)}\left[4 \lambda_{k}^{3}-\sum_{n=1}^{m}\left(2 \lambda_{k} p_{n} q_{n}+p_{n, x} q_{n}-p_{n} q_{n, x}\right)\right.
$$

$$
\left.\left.+\sum_{n=1}^{m} \varpi_{k}^{(n)}\left(4 \lambda_{k}^{2} p_{n}+2 \lambda_{k} p_{n, x}-2 \sum_{r=1}^{m} p_{r} q_{r} p_{n}+p_{n, x x}\right)\right]\right\} \beta_{j}\left(\lambda_{k}\right)-\sum_{j=1}^{m} \varpi_{k}^{(j)} \beta_{j, t}\left(\lambda_{k}\right),
$$

$$
\begin{aligned}
\gamma_{i, t}\left(\lambda_{k}\right)= & -\sum_{j=1}^{m}\left\{4 \lambda_{k}^{2} q_{j}-2 \lambda_{k} q_{j, x}-2 q_{j} \sum_{n=1}^{m} p_{n} q_{n}+q_{j, x x}-4 \lambda_{k}^{3} \varpi_{k}^{(j)}+2 \lambda_{k} q_{j} \sum_{n=1}^{m} p_{n} \varpi_{k}^{(n)}\right. \\
& -q_{j, x} \sum_{n=1}^{m} p_{n} \varpi_{k}^{(n)}+q_{j} \sum_{n=1}^{m} p_{n, x} \varpi_{k}^{(n)}-\varpi_{k}^{(j)}\left[4 \lambda_{k}^{3}-\sum_{n=1}^{m}\left(2 \lambda_{k} p_{n} q_{n}+p_{n, x} q_{n}\right.\right.
\end{aligned}
$$$$
\left.\left.\left.-p_{n} q_{n, x}\right)+\sum_{n=1}^{m} \varpi_{k}^{(n)}\left(4 \lambda_{k}^{2} p_{n}+2 \lambda_{k} p_{n, x}-2 \sum_{r=1}^{m} p_{r} q_{r} p_{n}+p_{n, x x}\right)\right]\right\} \delta_{i j}\left(\lambda_{k}\right)
$$

$$
-\sum_{j=1}^{m} \varpi_{k}^{(j)} \delta_{i j, t}\left(\lambda_{k}\right) \quad(1 \leq i \leq m) .
$$

By means of Eqs. (15) and (A.8)-(A.13), we remove $\alpha\left(\lambda_{k}\right), \gamma_{i}\left(\lambda_{k}\right), \alpha_{x}\left(\lambda_{k}\right), \gamma_{i, x}\left(\lambda_{k}\right), \alpha_{y}\left(\lambda_{k}\right), \gamma_{i, y}\left(\lambda_{k}\right)$, $\alpha_{t}\left(\lambda_{k}\right)$ and $\gamma_{i, t}\left(\lambda_{k}\right)$ from the right-hand sides of Eqs. (A.2)-(A.7), and finally prove that $u_{h l}\left(\lambda_{k}\right)=0$, $v_{h l}\left(\lambda_{k}\right)=0$ and $w_{h l}\left(\lambda_{k}\right)=0(1 \leq h, l \leq m+1)$. 


\section{References}

[1] B. B. Kadomtsev and V. I. Petviashvili, On the stability of solitary waves in weakly dispersing media, Sov. Phys. Doklady 15, 539-541 (1970).

[2] M. J. Ablowitz and H. Segur, On the evolution of packets of water waves, J. Fluid Mech. 92, 691-715 (1979).

[3] M. J. Ablowitz and P. A. Clarkson, Solitons, nonlinear evolution equations and inverse scattering, Cambridge Univ. Press, Cambridge, 1992.

[4] V. S. Dryuma, Analytic solution of the two-dimensional Korteweg-de Vries equation, Sov. Phys. JETP Lett. 19, 387-388 (1974).

[5] J. Satsuma and M. J. Ablowitz, Two-dimensional lumps in nonlinear dispersive systems, J. Math. Phys. 20, 1496-1503 (1979).

[6] J. Satsuma, $N$-soliton solution of the two-dimensional Korteweg-de Vries equation, J. Phys. Soc. Jpn. 40, 286-290 (1976).

[7] W. Oevel and B. Fuchssteiner, Explicit formulas for symmetries and conservation laws of the Kadomtsev-Petviashvili equation, Phys. Lett. A 88, 323-327 (1982).

[8] J. Weiss, M. Tabor and G. Carnevale, The Painlevé property for partial differential equations, J. Math. Phys. 24, 522-526 (1983).

[9] H. H. Chen, A Bäcklund transformation in two dimensions, J. Math. Phys. 16, 2382-2384 (1975).

[10] V. B. Matveev, Darboux transformation and explicit solutions of the Kadomtsev-Petviashvili equation, depending on functional parameters, Lett. Math. Phys. 3, 213-216 (1979).

[11] A. S. Fokas and P. M. Santini, The recursion operator of the Kadomtsev-Petviashvili equation and the squared eigenfunctions of the Schrödinger operator, Stud. Appl. Math. 75, 179-185 (1986).

[12] A. S. Fokas and P. M. Santini, Bi-Hamiltonian formulation of the Kadomtsev-Petviashvili and Benjamin-Ono equations, J. Math. Phys. 29, 604-617 (1988).

[13] M. Tajiri, T. Nishitani and S. Kawamoto, Similarity solutions of the Kadomtsev-Petviashvili equation, J. Phys. Soc. Jpn. 51, 2350-2356 (1982).

[14] D. David, D. Levi and P. Winternitz, Symmetry reduction for the Kadomtsev-Petviashvili equation using a loop algebra, J. Math. Phys. 27, 1225-1237 (1986).

[15] S. Y. Lou, Similarity solutions of the Kadomtsev-Petviashvili equation, J. Phys. A 23, L649-L654 (1990).

[16] G. Biondini and Y. Kodama, On a family of solutions of the Kadomtsev-Petviashvili equation which also satisfy the Toda lattice hierarchy, J. Phys. A 36, 10519-10536 (2003).

[17] G. Biondini and S. Chakravarty, Soliton solutions of the Kadomtsev-Petviashvili II equation, J. Math. Phys. 47, 033514:1-26 (2006).

[18] G. Biondini, Line soliton interactions of the Kadomtsev-Petviashvili equation, Phys. Rev. Lett. 99, 064103:1-4 (2007). 
[19] S. Chakravarty and Y. Kodama, Classification of the soliton solutions of KPII, J. Phys. A 41, 275209:133 (2008).

[20] S. Chakravarty and Y. Kodama, Soliton solutions of the KP equation and application to shallow water waves, Stud. Appl. Math. 123, 83-151 (2009).

[21] Y. Kodama, M. Oikawa and H. Tsuji, Soliton solutions of the KP equation with V-shape initial waves, J. Phys. A 42, 312001:1-9 (2009).

[22] Y. Kodama, KP solitons in shallow water, J. Phys. A 43, 434004:1-54 (2010).

[23] C. Y. Kao and Y. Kodama, Numerical study of the KP equation for non-periodic waves, Math. Comput. Simul., in Press, 2011.

[24] N. C. Freeman and J. J. C. Nimmo, Soliton-solutions of the Korteweg-de Vries and KadomtsevPetviashvili equations: the Wronskian technique, Phys. Lett. A 95, 1-3 (1983).

[25] G. Biondini and T. Xu, Irreducible, totally non-negative Grassmann cells, irreducible Le-diagrams and derangements, submitted.

[26] A. Postnikov, Total positivity, Grassmannians, and networks, Preprint math.CO/0609764 (2006).

[27] B. Konopelchenko, J. Sidorenko and W. Strampp, (1+1)-dimensional integrable systems as symmetry constraints of (2+1)-dimensional systems, Phys. Lett. A 157, 17-21 (1991).

[28] Y. Cheng and Y. S. Li, The constraint of the Kadomtsev-Petviashvili equation and its special solutions, Phys. Lett. A 157, 22-26 (1991).

[29] T. Xu and B. Tian, An extension of the Wronskian technique for the multicomponent Wronskian solution to the vector nonlinear Schrödinger equation, J. Math. Phys. 51, 033504:1-21 (2010).

[30] T. Xu, B. Tian, Y. S. Xue and F. H. Qi, Direct analysis of the bright-soliton collisions in the focusing vector nonlinear Schrödinger equation, EPL 92, 50002:1-5 (2010).

[31] T. Xu, B. Tian and F. H. Qi, Bright $N$-soliton solution to the vector Hirota equation from nonlinear optics with symbolic computation, submitted to Zeitschrift für Naturforschung A.

[32] C. H. Gu, H. S. Hu and Z. X. Zhou, Darboux transformation in soliton theory and its geometric applications, Shanghai Sci.-Tech. Pub., Shanghai, 2005.

[33] Q. P. Liu, Double Wronskian solutions of the AKNS and the classical Boussinesq hierarchies, J. Phys. Soc. Jpn. 59, 3520-3527 (1990).

[34] M. J. Ablowitz and R. Haberman, Resonantly coupled nonlinear evolution equations, J. Math. Phys. 16, 2301-2305 (1975).

[35] A. P. Fordy and P. P. Kulish, Nonlinear Schrödinger equations and simple Lie algebras, Commun. Math. Phys. 89, 427-443 (1983).

[36] T. Tsuchida and M. Wadati, The coupled modified Korteweg-de Vries equations, J. Phys. Soc. Jpn. 67, 1175-1187 (1998).

[37] E. Infeld and G. Rowlands, Nonlinear waves, solitons and chaos, Cambridge Univ. Press, Cambridge, 1990. 
[38] M. Haelterman and A. Sheppard, Bifurcation phenomena and multiple soliton-bound states in isotropic Kerr media, Phys. Rev. E 49, 3376-3381 (1994). 